\title{
INFINITE-DIMENSIONAL GROUP REPRESENTATIONS ${ }^{1}$
}

\author{
GEORGE W. MACKEY
}

1. Introduction. Let $S$ be a set in which there is given a $\sigma$-field of "Borel sets" and let $G$ be a topological group. Let $s x$ be defined for all $s$ in $S$ and all $x$ in $G$ in such a manner that

(a) $s x_{1} x_{2}=\left(s x_{1}\right) x_{2}$,

(b) $s e=s$,

(c) $s, x \rightarrow s x$ is a Borel function

from $S \times G$ to $S$. Under these circumstances we shall say that $S$ is a Borel $G$ space. We shall usually assume that $S$ is standard in the sense that it is isomorphic as a Borel space to a Borel subset of a separable complete metric space. Let $\mu$ be a Borel measure in $S$, that is, a $\sigma$-finite countably additive measure defined on all Borel subsets of $S$. If $\mu(E x)=\mu(E)$ for all $E$ we shall say that $\mu$ is invariant. Given an invariant $\mu$ in the Borel space $S$ we may form the Hilbert space $\mathcal{L}^{2}(S, \mu)$ and observe that for each $x$ in $G$ the operator $L_{x}$ such that $\left(L_{x}(f)\right)(s)=f(s x)$ is a unitary operator. Moreover $x \rightarrow L_{x}$ is a unitary representation of the group $G$ in a sense to be made precise below. When $S=G, G$ is locally compact and $s x$ is group multiplication, the measure $\mu$ exists and is essentially unique. The unitary representation $L$ in this case is called the regular representation of $G$.

Consider the special case in which $G$ is the compact group $K$ of all rotations in the plane-or equivalently the group obtained from the additive group of the real line by identifying numbers which differ by integral multiples of $2 \pi$.

The functions $e^{i n x}(n=0, \pm 1, \pm 2, \cdots)$ form a basis for $\mathfrak{L}^{2}(K)$ and each member of this basis generates a one-dimensional subspace which is invariant under each $L_{x}$. We have, in a sense to be defined below, a decomposition of the regular representation of $K$ into ir-

The preparation of this paper was supported in part by the National Science Foundation under grant NSF-G 16428 to the American Mathematical Society; received by the editors May 6, 1963.

${ }^{1}$ Except for the appendix this paper constitutes the text of the "notes" distributed in pamphlet form to the audience at the author's Colloquium Lectures delivered during the 1961 summer meeting of the American Mathematical Society in Stillwater, Oklahoma. These notes were written earlier the same summer while the author was a Walker Ames visiting professor of Mathematics at the University of Washington. In the original version the bibliography was included informally in the text of $\$ 11$. The supplementary bibliography was written to accompany the appendix. 
reducible parts. The fact that this decomposition exists is a simple reformulation of one of the fundamental theorems in the theory of Fourier series. The celebrated Plancherel formula for Fourier transforms provides a similar decomposition for the regular representation of the additive group of the real line. However here one has a continuous decomposition into "infinitesimal" parts.

The theory we are going to describe in these lectures begins with and is to some extent dominated by attempts to answer the following two questions.

(a) To what extent can an arbitrary unitary representation of a group be analyzed into irreducible ones.

(b) Given a group $G$ to what extent can one find all possible irreducible representations of $G$.

Such answers as are available represent extensions of classical harmonic analysis in various directions and include a large part of what group theory has contributed to analysis. The particular representations defined by invariant measures in Borel $G$ spaces are much more general than they at first appear to be and certain natural generalizations of them come close to being exhaustive. Certainly they are the most important examples occurring naturally in analysis and physics.

When $G$ is finite the theory is purely algebraic in character and (barring classical harmonic analysis whose group theoretical character was clearly recognized only much later) it is with this case that the subject begins. Started by Frobenius in 1900 it was dominated by the names of Frobenius and Schur for the next decade or so. In the mid 1920's it was extended to compact Lie groups by Schur, Weyl and others. The celebrated Peter-Weyl theorem says that the regular representation of a compact Lie group is a direct sum of finitedimensional irreducible representations. With A. Haar's (1933) discovery of the existence of an invariant measure on any locally compact group it was realized that the Peter-Weyl theorem and much of the rest of the theory applies to arbitrary compact groups. When the compactness hypothesis is discarded there are serious difficulties due to the fact that one may have to deal with continuous decompositions and infinite-dimensional irreducible components. The second difficulty disappears when the group is commutative for then all irreducible representations are one-dimensional and one obtains a close analogue of the theory of the Fourier transform. This theory is based in part on the Pontrjagin-van Kampen duality theory for locally compact groups which was developed in the mid 1930's.

Insofar as unitary representations of compact and commutative groups are concerned the theory was well nigh complete in 1938 and 
is described in detail in A. Weil's classic treatise. We shall be chiefly concerned with the post World War II theory in which one must deal simultaneously with continuous decompositions and infinite-dimensional irreducible constituents. Although there was an important paper by Wigner in 1939, another by Gelfand and Raikov in 1943 and a few preliminary announcements by Gelfand and Naimark in 1946, this part of the theory did not really get under way until 1947 when papers began to appear at the rate of five to ten a year.

In the fourteen years which have elapsed since this deluge began the theory has developed far too much to be summarized adequately in ten lectures-let alone four. Since we must select we hope we may be excused for emphasizing those parts of the theory with which we have been most directly concerned. Actually we have reason to believe that a fairly large section of the theory will ultimately be fitted into the framework which we shall outline here.

2. Some basic notions. We shall deal throughout with topological groups $G$ which are locally compact and are separable in the sense that there exists a countable basis for the open sets. By a unitary representation $L$ of such a group $G$ we shall mean a mapping $x \rightarrow L_{x}$ from $G$ to the unitary operators in some separable Hilbert space $3 C(L)$ which has the following two properties:

(a) $L_{x y}=L_{x} L_{y}$ for all $x$ and $y$ in $G$.

(b) $x \rightarrow L_{x}(\phi)$ is a continuous function from $G$ to $\mathfrak{H C}(L)$ for all $\phi$ in $\operatorname{HC}(L)$.

It is known that property (b) is implied by the ostensibly much weaker property $\left(b^{\prime}\right) x \rightarrow\left(L_{x}(\phi), \psi\right)$ is measurable with respect to Haar measure in $G$ for all $\phi$ and $\psi$ in $\operatorname{HC}(L)$.

By an intertwining operator for $L$ and $M$ we shall mean a bounded linear operator $T$ from $\mathfrak{F C}(L)$ to $\mathfrak{H C}(M)$ such that $T L_{x}=M_{x} T$ for all $x$ in $G$. The set of all such is a vector space which we denote by $\Re(L, M)$. When $L=M, \Re(L, M)=\Re(L, L)$ is a (weakly closed) subalgebra of the algebra of all bounded linear operators on $\mathcal{H}$. It is called the commuting algebra of $L$. If $R(L, M)$ contains a unitary map $U$ of $\mathfrak{H C}(L)$ onto $\mathfrak{H C}(M)$ then $U L_{x} U^{-1}=M_{x}$ and $L$ and $M$ are said to be equivalent representations of $G$. We shall write $L \simeq M$. It is seldom necessary to distinguish between equivalent representations.

If $L^{1}, L^{2}, \ldots$ are unitary representations of $G$ we define their direct sum $L=L^{1} \oplus L^{2} \oplus \cdots$ as follows. $\Re(L)$ is the direct sum of the Hilbert spaces $\mathfrak{F C}\left(L^{1}\right), \mathfrak{C}\left(L^{2}\right), \cdots$, i.e. the set of all sequences $\phi^{1}, \phi^{2}, \cdots$ with $\left\|\phi^{1}\right\|^{2}+\left\|\phi^{2}\right\|^{2}+\cdots<\infty, \phi^{j} \in \mathcal{H}\left(L^{j}\right)$ and the obvious definition of the Hilbert space operations. $L_{x}\left(\phi^{1}, \phi^{2}, \cdots\right)=L_{x}\left(\phi^{1}\right)$, $L_{x}\left(\phi^{2}\right), \cdots$. 
Let $\mathfrak{H}_{1}$ be a closed subspace of $\mathfrak{F}(L)$. If $L_{x}(\phi) \in \mathfrak{K}_{1}$ for all $x \in G$ and all $\phi \in \mathfrak{F C}_{1}$ we say that $\mathfrak{H C}_{1}$ is an invariant subspace of $\mathfrak{H C}(L)$. If $L_{x}^{\mathfrak{F C}}$ denotes the restriction of $L_{x}$ to $\mathfrak{F C}_{1}$ then $x \rightarrow L_{x}^{\mathfrak{F}_{1}}$ is a unitary representation of $G$, which we call the subrepresentation defined by $\mathfrak{F C}_{1}$. If $P$ is the unique projection whose range is $\mathfrak{F C}_{1}$ then $\mathcal{F C}_{1}$ is invariant if and only if $P \in R(L, L)$ so that the subrepresentations of $L$ correspond one-to-one to the projections in $R(L, L)$. We shall use $L^{P}$ as an alternative notation for $L^{\mathfrak{F C}_{1}}$. It is easy to see that $L$ is equivalent to $L^{P} \oplus L^{1-P}$. Thus the following statements about $L$ are equivalent:

(a) $L$ is not equivalent to a (nontrivial) direct sum.

(b) $R(L, L)$ contains no nontrivial projections.

(c) $L$ has no (nontrivial) subrepresentations.

Using the spectral theorem (b) can be shown to be equivalent to

$\left(b^{\prime}\right) \quad R(L, L)$ contains only scalar multiples of the identity $I$.

When any one and hence all of these equivalent statements hold $L$ is said to be irreducible.

Let there exist a nonzero member $T$ of $R(L, M)$. Let $N_{T}$ denote the set of all $\phi$ with $T(\phi)=0$ and let $\bar{W}_{T}$ denote the closure of the set of all $T(\phi)$. It is immediate that $N_{T}$ and $\bar{W}_{T}$ are invariant and it follows from an extension of a classical lemma of Schur that the subrepresentation of $L$ defined by $N_{T}^{\frac{1}{T}}$ is equivalent to the subrepresentation of $M$ defined by $\bar{W}_{T}$. Thus $R(L, M)$ reduces to 0 if and only if no subrepresentation of $L$ is equivalent to any subrepresentation of $M$. When this is so we say that $L$ and $M$ are disjoint and write $L \delta M$. To pass to the opposite extreme we say that $L$ and $M$ are quasi equivalent if no (nonzero) subrepresentation of $L$ is disjoint from $M$ and no (nonzero) subrepresentation of $M$ is disjoint from $L$. For example if $L$ is irreducible then $L \oplus L$ is quasi equivalent but not equivalent to $L$. Moreover two inequivalent irreducible representations are always disjoint.

It is not hard to show that $L^{P}$ and $L^{1-P}$ are disjoint if and only if $P$ is in the center of $R(L, L)$. When this center reduces to multiples of the identity so that it is impossible to decompose $L$ as a direct sum of disjoint parts we say that $L$ is primary. When the center is all of $\mathbb{R}(L, L)$ so that $\mathcal{R}(L, L)$ is commutative and every decomposition of $L$ as a direct sum has disjoint parts we say that $L$ is multiplicity free.

If $L=L^{1} \oplus L^{2} \oplus \ldots$ where the $L^{i}$ are all irreducible then $L$ is primary if and only if $L^{i} \simeq L^{j}$ for all $i$ and $j$ and $L$ is multiplicity free if and only if $L^{i} \simeq L^{i}$ implies $i=j$.

If $n=1,2, \cdots, \aleph_{0}$ then $n L$ will denote the direct sum $L \oplus L \oplus \cdots$ taken to $n$ terms. If $\boldsymbol{\aleph}_{0} L \simeq L$ we shall say that $L$ is infinite. Clearly $\aleph_{0} L$ is infinite whenever $L \neq 0$. If no subrepresentation of $L$ is infinite 
we shall say that $L$ is finite. If $L$ is neither finite nor infinite there exists a unique projection $P_{f}$ in the center of $R(L, L)$ such that $L^{P f}$ is finite and $L^{1-P_{f}}$ is infinite. Of course $L^{P_{f}} \delta L^{1-P_{f}}$.

Let $L=n L^{0}$ where $L^{0}$ is irreducible. Then $L$ is primary and has an irreducible subrepresentation. Conversely if $L$ is primary and has an irreducible subrepresentation $L^{0}$ then $L \simeq n L^{0}$ for some $n=1,2, \cdots$, $\aleph_{0}$. Any two irreducible subrepresentations $L^{0}$ and $L^{00}$ of $L$ are equivalent and $n L^{0} \simeq n^{\prime} L^{00}$ implies $n=n^{\prime}$ and $L^{0} \simeq L^{00} . n=n^{\prime}$ is called the multiplicity of $L$. A primary representation with an irreducible subrepresentation is said to be of type I. There are two further possibilities. It may be that, though $L$ is not irreducible, every proper subrepresentation of $L$ is equivalent to $L$. Such a representation is necessarily primary and infinite and is said to be primary of type III. A primary representation which has a finite subrepresentation but no irreducible subrepresentation is said to be of type II. If $L$ is finite, primary and of type II it may always be "cut in half"; that is there exists a primary subrepresentation $L^{1}$ such that $L \simeq L^{1} \oplus L^{1}$ and $L^{1}$ is unique to within equivalence. By iteration $\left(1 / 2^{k}\right) L$ is defined to within equivalence for all $k=1,2, \cdots$. Thus we may define $\lambda L$ where $\lambda$ is any positive real number by writing $\lambda$ in the form $n+1 / 2^{k_{1}}+1 / 2^{k_{2}}+1 / 2^{k_{3}}+\cdots$ and setting $\lambda L=n L+\left(1 / 2^{k_{1}}\right) L$ $+\left(1 / 2^{k_{2}}\right) L+\cdots$. It can be shown that each $\lambda L$ is finite, primary and quasi equivalent to $L$ and that every representation quasi equivalent to $L$ is either finite and equivalent to a unique $\lambda L$ or else infinite and equivalent to $\boldsymbol{\aleph}_{0} L$. It follows that the set of all representation quasi equivalent to a given primary representation has a natural ordering which is either that of the positive integers and $\boldsymbol{\aleph}_{0}$, the positive reals and $\boldsymbol{\aleph}_{0}$, or $\boldsymbol{\aleph}_{0}$ alone.

If $L=L^{1} \oplus L^{2} \oplus \cdots$ where the $L^{i}$ are disjoint and primary then the following statements are easily proved.

(a) Each $L^{j}$ is of type $I$ if and only if $L$ is quasi equivalent to a multiplicity free representation.

(b) Each $L^{i}$ is of type III if and only if every representation quasi equivalent to $L$ is equivalent to $L$.

(c) Each $L^{j}$ is of type II if and only if $L$ is quasi equivalent to a finite representation and every representation quasi equivalent to $L$ is of the form $M \oplus M$ for some $M$.

Thus it is natural to make the following definitions. A (not necessarily primary) representation $L$ is of type $I$ if it is equivalent to a multiplicity free representation. It is of type III if every representation quasi equivalent to $L$ is equivalent to $L$. It is of type II if it is quasi equivalent to a finite representation and every representation 
quasi equivalent to $L$ is of the form $M \oplus M$ for some $M$. It can be shown that for any $L$ there exist unique projections $P^{\mathrm{I}}, P^{\mathrm{II}}$, and $P^{\mathrm{III}}$ in the center of $R(L, L)$ such that

(a) $L^{P^{j}} \delta L^{P^{k}}$ for $j \neq k$,

(b) $L \simeq L^{P^{\mathrm{I}}} \oplus L^{P^{\mathrm{II}}} \oplus L^{P^{\mathrm{III}}}$,

(c) $L^{P^{i}}$ is of type $j$ whenever $P^{j} \neq 0$.

3. The decomposition of representations. Let us say that $L$ is discretely decomposable if $L \simeq L^{1} \oplus L^{2} \oplus \ldots$ where the $L^{j}$ are irreducible. If $M$ is irreducible then the dimension of $R\left(M, L^{1} \oplus L^{2} \oplus \cdots\right)$ is equal to the number of indices $k$ for which $M \simeq L^{k}$. We shall call this number the multiplicity of $M$ in $L^{1} \oplus L^{2} \oplus \ldots$. It is clear that $L$ is uniquely determined by the non-negative integers which are the dimensions of the spaces $R(M, L)$ whenever $L$ is discretely decomposable. Thus to know all irreducible unitary representations (to within equivalence) is to know all discretely decomposable unitary representations to within equivalence. When $G$ is compact every unitary representation is discretely decomposable but for more general groups discretely decomposable representations are rather special. While it is true that every unitary representation is (in a sense to be defined below) a direct integral of irreducible representations, this decomposition is too nonunique to be useful in the general case in reducing the study of unitary representations to that of irreducible ones. On the other hand there are many important noncompact groups which are known to have only type I representations. For these the direct integral decomposition into irreducibles is essentially unique and there is a sense in which finding the general unitary representation can be reduced to finding the general irreducible unitary representation-both to within equivalence of course. When type II and type III representations exist there is a somewhat weaker sense in which finding the most general unitary representation can be reduced to finding the most general quasi equivalence class of primary representations. However there is no case known in which one has nontype I representations and can describe (in any reasonable sense) the set of all equivalence classes of irreducibles-let alone the set of all quasi equivalence classes of primary representations.

Let $S$ be a standard Borel space and let $\mu$ be a finite measure in $S$. Let there be given a Hilbert space $\mathfrak{H C}$ and for each $s \in S$ let $L^{z}$ be a unitary representation of our group $G$. Let $L^{s}$ be a Borel function of $s$ in the sense that $\left(L_{x}^{s}(\phi) \cdot \psi\right)$ is a complex-valued Borel function on $S \times G$ for all $\phi$ and $\psi$ in $\Re$. We then define a new unitary representation $L$ as follows. $\mathfrak{F C}(L)=\mathscr{L}^{2}(S, \mu, \mathfrak{F C})$; that is, the Hilbert space of all Fe-valued square summable functions on $S$. For each $x \in G$ and each 
$g \in \mathscr{L}^{2}(S, \mu, \mathcal{F}), L_{x}(g)(s)=L_{x}^{s}(g(s))$. We call $L$ the direct integral of the $L^{s}$ with respect to $L^{s}$ and denote it by $\int_{S} L^{s} d \mu(s)$. [If the $L^{s}$ are given to us as acting in different Hilbert spaces $\mathfrak{H}^{s}$ or as equivalence classes of representations then we may make the above construction whenever it is possible to map all $\mathrm{HC}^{\mathrm{B}}$ of a given dimension onto a single Hilbert space in such a manner that the above condition on $L^{*}$ is satisfied. It may be shown that the equivalence class of $\int_{S} L^{s} d \mu(s)$ does not depend upon which mappings are chosen and that the mappings exist if and only if the Hilbert space "bundle" of all pairs $s, \phi$ with $\phi \in \mathcal{H C}_{s}$ can be made into a Borel space having certain simple natural properties. In practice there is often a "natural" Borel structure in the bundle of pairs $s, \phi$.]

Let $\nu$ be a second measure in $S$ having the same null sets as $\mu$. Let $\rho$ be the Radon-Nikodym derivative of $\nu$ with respect to $\mu$. Then $f \rightarrow \sqrt{ }(\rho) f$ defines a unitary map of $\mathscr{L}^{2}(S, \nu, \mathfrak{F C})$ on $\mathscr{L}^{2}(S, \mu, \mathfrak{F})$ which sets up an equivalence between $\int_{S} L^{s} d \nu(s)$ and $\int_{S} L^{s} d \mu(s)$. In other words in forming direct integrals it is only the family of null sets which counts-not the measure itself. It is even possible to redefine $\int_{S} L^{*} d \mu(s)$ in such a way as to avoid choosing a particular $\mu$ but we shall not give details here. It is obvious that $\int_{S^{\prime}} L^{s} d \mu(s) \simeq \int_{S} L^{s} d \mu(S)$ whenever $S^{\prime} \subseteq S$ and $\mu\left(S-S^{\prime}\right)=0$ and it is true but not obvious that $\int_{S} L^{s} d \mu(s)$ $\simeq \int_{S} M^{s} d \mu(s)$ if $L^{s} \simeq M^{s}$ for almost all $s$.

For each Borel subset $E$ of $S$ let $P_{E}$ denote the projection in $\mathscr{L}^{2}(S, \mu, \mathcal{F})$ which takes $f$ into $\phi_{E} f$ where $\phi_{E}$ is the characteristic function of $E$. Then $E \rightarrow P_{E}$ is a projection-valued measure; that is a function from Borel sets to projections having the following three properties:

(a) $P_{0}=0$ and $P_{S}=I$ ( $I$ is the identity operator),

(b) $P_{E \cap F}=P_{E} P_{F}$,

(c) if $E_{i} \cap E_{j}=0$ for $i \neq j$ then $P_{\left(E_{1} \cup F_{2} \cup \cdots\right)}=P_{E_{1}}+P_{E_{2}}+\cdots$.

Moreover each $P_{E}$ is in $R(L, L)$. Conversely let $L^{\prime}$ be any unitary representation of $G$ and let $P^{\prime}$ be a projection-valued measure defined on the Borel subsets of a standard Borel space $S$ such that $P_{E}^{\prime}$ is in $\mathbb{R}(L, L)$ for all $E$. Then there exists an assignment $s \rightarrow L^{s}$ of a unitary representation to each $s$ in $S$ and a finite measure $\mu$ in $S$ such that $L=\int L^{s} d \mu(s)$ exists and is equivalent to $L^{\prime}$. In addition the unitary mapping setting up the equivalence may be chosen so that it carries $P^{\prime}$ into the projection-valued measure $P$ associated with the direct integral $\int L^{s} d \mu(s)$ in the manner described above. The null sets of $\mu$ are just the sets $E$ such that $P_{E}^{\prime}=0$ and to within equivalence $L^{s}$ is uniquely determined for almost all $s$. Thus a representation $L$ together with a projection-valued measure $P$ such that $P_{E} L_{x}=L_{x} P_{E}$ for 
all $E$ and $x$ is essentially the same thing as a representation $L$ together with a particular realization of $L$ as a direct integral.

Actually the space $S$ can be eliminated from the above. Let $P^{1}$ and $P^{2}$ be projection-valued measures defined on standard Borel spaces $S_{1}$ and $S_{2}$. Suppose that the range of $P^{1}$ is identical with the range of $P^{2}$, i.e. that every $P_{E}^{1}$ is equal to some $P_{E}^{2}$ and vice versa. Then there exists a one-to-one map $\theta$ of $S_{1}$ on $S_{2}$ such that $E$ is a Borel set if and only if $\theta(E)$ is a Borel set and such that $P_{\theta(E)}^{1}=P_{E}^{2}$ for all $E$. Let $F$ be a family of projections in the (separable) Hilbert space JC. Then $\mathcal{F}$ is the range of a projection-valued measure $P$ defined on a standard Borel space if and only if it has the following properties:

(a) $Q_{1} Q_{2}=Q_{2} Q_{1} \in \mathcal{F}$ whenever $Q_{1} \in \mathcal{F}$ and $Q_{2} \in \mathcal{F}$.

(b) If $Q \in \mathcal{F}$ then $1-Q \in F$.

(c) If $Q_{1}, Q_{2}, \cdots \in \mathcal{F}$ and $Q_{i} Q_{j}=0$ for $i \neq j$ then $Q_{1}+Q_{2}$ $+\cdots \in F$.

A family $\mathcal{F}$ having these three properties is called a $\sigma$ Boolean algebra of projections.

It follows from the above that there is a natural one-to-one correspondence between the representations of a given $L$ as a direct integral and the different $\sigma$ Boolean algebras of projections in $\Re(L, L)$. Let $\int_{S} L^{s} d \mu(s)$ be the essentially unique direct integral decomposition of $L$ associated with the $\sigma$ Boolean algebra of projections $\mathcal{F}$ in $R(L, L)$. One knows the following about the connections between $F$ and the $L^{s}$.

(a) Almost every $L^{8}$ is irreducible if and only if $F$ is maximal in R $(L, L)$.

(b) Almost every $L^{s}$ is primary if and only if $\mathcal{F}$ contains the center of $R(L, L)$.

(c) If $\mathcal{F}$ is equal to the center of $R(L, L)$ then there exists a subset $N$ of $S$ such that $\mu(N)=0$ and such that $L^{s} \delta L^{8^{\prime}}$ if $s \in S-N$ and $s^{\prime} \in S-N$.

It follows from (a) and Zorn's lemma that every $L$ has a direct integral decomposition into irreducible parts. This decomposition may vary greatly from one maximal $F$ to another when $L$ is not of type I. For example there exists a type II primary representation and two direct integral decompositions $L \simeq \int_{S} L^{s} d \mu(s), L \simeq \int_{S^{\prime}} L^{\prime t} d \nu(t)$ such that no $L^{s}$ is equivalent to any $L^{\prime t}$ and such that each $L^{s}$ is irreducible and each $L^{\prime t}$ is irreducible.

The decomposition into disjoint primary components defined by taking $F$ to be all projections in the center of $R(L, L)$ is of course canonical and is called the central decomposition of $L$.

When $L$ is multiplicity free the set of all projections in the center 
of $\mathcal{Q}(L, L)$ is the set of all projections in $R(L, L)$ and hence is maximal. Thus, in this case, the central decomposition of $L$ is into inequivalent irreducible parts. Let $\hat{G}$ denote the set of all equivalence classes of irreducible representations of $G$. Let $L$ be multiplicity free and let $\int L^{s} d \mu(s)$ denote the central decomposition chosen so that the $L^{s}$ are all irreducible. Then the mapping $s \rightarrow L^{s}$ defines a mapping of $S$ into $\hat{G}$ and takes $\mu$ into a measure $\hat{\mu}$ in $\hat{G}$. The null sets of $\hat{\mu}$ are independent of the arbitrary features in $\int_{S} L^{s} d \mu(s)$ so that each multiplicity free $L$ defines a unique measure class $C_{L}$ which in turn determines $L$ to within equivalence. It is possible to define the notion of Borel set in $\hat{G}$ in a natural manner and when this is done the measure class $C_{L}$ turns out to be a Borel measure class. However if $G$ has nontype I representations there may exist Borel measure classes in $\hat{G}$ which do not arise from any multiplicity free representation. A fairly interesting unsolved problem is that of characterizing intrinsically the measure classes which arise from multiplicity free representations. On the other hand it is known that $\hat{G}$ is a standard Borel space if and only if every representation of $G$ is of type $I$. When either and hence both of these conditions hold, every Borel measure class in $\hat{G}$ comes from a multiplicity free representation and we have a natural one-toone correspondence between the measure classes and the equivalence classes of multiplicity free representations. Now the study of type I representations can be reduced (without using direct integral theory) to the study of multiplicity free representations. Hence for type I groups (defined as those having only type I representations) the study of the general unitary representation can be reduced to the study of measure classes in $\hat{G}$-and thus to the determination of $\hat{G}$ and a classical problem in real variable theory.

The "type I to multiplicity free" reduction is a consequence of the following theorem. Let $L$ be any type I representation. Then there exist disjoint multiplicity free representations $L^{\infty}, L^{1}, L^{2}, \ldots$ uniquely determined by $L$ to within equivalence such that $L \simeq \boldsymbol{\aleph}_{0} L^{\infty} \oplus L^{1} \oplus 2 L^{2} \oplus \ldots$. Of course some of the $L^{i}$ may be missing.

Amongst the separable locally compact groups which are known to have only type I representations are the compact groups, the commutative groups, the connected semisimple Lie groups and the connected real algebraic groups. However there are many examples of connected solvable Lie groups which have nontype I representations.

It is known that the regular representation of a discrete group is of type II whenever the subgroup generated by the finite conjugate classes has infinite index. Moreover it is known that a countable restricted direct product of finite groups always has a type II regular 
representation. No examples are known of type I discrete groups except for finite extensions of commutative groups.

4. Characters, spherical functions and the Plancherel formula. For any Hilbert space let $J$ denote the canonical antilinear mapping of $\mathcal{H C}$ on the Banach space adjoint $\overline{\mathcal{K}}$ of $\mathcal{H C}$ and if $T$ is a bounded linear operator from $\mathfrak{F C}_{1}$ to $\mathfrak{H C}_{2}$ let $T^{0}$ denote the Banach space adjoint from $\overline{\mathfrak{F}}_{2}$ to $\overline{\mathfrak{K}}_{1}$. Then the ordinary Hilbert space adjoint $T^{*}$ from $\mathfrak{F C}_{2}$ to $\mathfrak{F C}_{1}$ will be equal to $J T^{0} J$. For each unitary representation $L$ the mapping $x \rightarrow L_{x^{-1}}^{0}$ is a unitary representation which we shall call the adjoint of $L$ and denote by $\bar{L}$. The set of all bounded linear operators $T$ from $\overline{\mathscr{F}}_{2}$ to $\mathfrak{F C}_{1}$ such that Trace $\left(T T^{*}\right)<\infty$ is a Hilbert space under the inner product $\left(T_{1}: T_{2}\right)=\operatorname{Trace}\left(T_{1} T_{2}^{*}\right)$. It is called the Kronecker or tensor product of $\mathfrak{F C}_{1}$ with $\mathfrak{F C}_{2}$ and denoted by $\mathfrak{H C}_{1} \otimes \mathfrak{K C}_{2}$. If $A_{1}$ and $A_{2}$ are bounded linear operators on $\mathfrak{F C}_{1}$ and $\mathfrak{F C}_{2}$ respectively, then $T \rightarrow A_{1} T A_{2}^{0}$ is a bounded linear operator $A_{1} \times A_{2}$ in $\mathfrak{H C}_{1} \otimes \mathfrak{H C}_{2}$. If $L^{1}$ and $L^{2}$ are unitary representations of $G_{1}$ and $G_{2}$ respectively, then $x, y \rightarrow L_{x}^{1} \times L_{y}^{2}$ is a unitary representation of $G_{1} \times G_{2}$ called the Kronecker or tensor products $L^{1} \times L^{2}$ of $L^{1}$ and $L^{2} . L^{1} \times L^{2}$ is irreducible whenever $L^{1}$ and $L^{2}$ are irreducible.

Let $a(G)$ denote the set of all set functions of the form $c_{1} \alpha_{1}+\ldots$ $+c_{n} \alpha_{n}$ where the $\alpha_{j}$ are finite Borel measures on $G$ and the $c_{j}$ are complex constants. $\alpha(G)$ is an algebra under convolution $\alpha, \beta \rightarrow \alpha \beta$ where $\int f(x) d(\alpha \beta)(x)=\int f(x y) d \alpha(x) d \beta(y)$ for all continuous $f$ with compact support. If $L$ is a unitary representation of $G$ and $\alpha \in Q(G)$ then $L_{\alpha}$ is defined to be the unique bounded linear operator such that $\left(L_{\alpha}(\phi) \cdot \psi\right)=\int\left(L_{x}(\phi) \cdot \psi\right) d \alpha(x)$ for all $\phi$ and $\psi$ in $\mathcal{F}(L) . \alpha \rightarrow L_{\alpha}$ is a homomorphism of $Q(G)$ into the ring of all bounded linear operators in $\operatorname{FC}(L)$. Moreover $L_{\alpha^{*}}=L_{\alpha}^{*}$ where $\alpha^{*}(E)=\left[\alpha\left(E^{-1}\right)\right]-{ }^{2}$ If $L$ is finite-dimensional $x \rightarrow \operatorname{Trace}\left(L_{x}\right)$ is a complex-valued function $x^{L}$ on $G$ called the character of $L . L$ is uniquely determined to within equivalence by its character $x^{L} \cdot \int x^{L}(x) d \alpha(x)=\operatorname{Trace}\left(L_{\alpha}\right)$ and $x^{L}$ determines and is determined by the linear functional $\alpha \rightarrow \operatorname{Trace}\left(L_{\alpha}\right)$ on $\alpha(L)$. Let $L=n_{1} L^{1} \oplus n_{2} L^{2} \oplus \cdots \oplus n_{k} L^{k}$ where the $L^{j}$ are irreducible and finitedimensional. Let us define an inner product in $\alpha(G)$ by setting $(\alpha: \beta)_{x_{L}}=\operatorname{Trace}\left(L_{\alpha \beta^{*}}\right)$. If we identify $\alpha$ with $\alpha^{\prime}$ whenever $\left(\alpha-\alpha^{\prime}: \alpha-\alpha^{\prime}\right)_{\Upsilon_{L}}=0, a(G)$ becomes a finite-dimensional Hilbert space and right and left translation in $Q(G)$ define unitary operators in this Hilbert space. Let $V^{X_{L}}$ denote the corresponding unitary

${ }^{2}$ Here and elsewhere the notation [ ] is used to avoid putting a bar over a group of symbols. According to the context it means either the conjugate of a complex number or the dual of a Banach space. 
representation of $G \times G$. The mapping $f \rightarrow L_{f}$ is a unitary member of $\mathcal{R}\left(V^{\mathfrak{X}_{L}}, L \times \bar{L}\right)$ and hence sets up an equivalence between $V^{X_{L}}$ and a subrepresentation of $L \times \bar{L}$. This representation is easily seen to be the multiplicity free representation $L_{1} \times \bar{L}_{1} \oplus L_{2} \times \bar{L}_{2} \oplus \cdots \oplus L_{k} \times \bar{L}_{k}$ whose restriction to $G \times e$ is quasi equivalent to $L$. If $x_{L}$ is replaced by $X=\lambda_{1} X^{L_{1}} \oplus \cdots \oplus \lambda_{k} X^{L_{k}}$ where the $\lambda_{j}$ are arbitrary positive real numbers $V^{X}$ may be defined just as $V^{X_{L}}$ was and is equivalent to $V^{X_{L}}$. However $f \rightarrow L_{f}$ is no longer unitary.

The above considerations may be extended to infinite-dimensional representations - but only to some and at the cost of defining a character to be an abstract linear functional defined on a proper subspace of $Q(G)$ and not necessarily associated with a point function on $G$. Specifically let us say that the unitary representation $L$ has a character if Trace $\left(L_{\alpha}\right)$ exists for some nonzero $\alpha$ in $Q(G)$. Let $Q^{L}(G)$ denote the set of all $\alpha$ for which Trace $\left(L_{\alpha}\right)$ exists and let $F_{L}$ denote the linear functional on $Q^{L}(G): \alpha \rightarrow \operatorname{Trace}\left(L_{\alpha}\right)$. We note that $Q^{L}(G)$ is always an ideal in $Q(G)$ and in particular is invariant under right and left translation.

We call $F_{L}$ the character of $L$. Given $F_{L}$ we define an inner product in $Q^{L}(G)$ by setting $(\alpha: \beta)_{F_{L}}=F_{L}\left(\alpha \beta^{*}\right)$ and convert $Q^{L}(G)$ into a preHilbert space by identifying as above. Left and right translations in the completion of this Hilbert space define a representation of $G \times G$ which we shall denote by $V^{F_{L}} . f \rightarrow L_{f}$ is a unitary member of $\mathcal{R}\left(V^{F_{L}}, L \times \bar{L}\right)$ and if $L$ is irreducible $f \rightarrow L_{f}$ sets up an equivalence between $V^{F_{L}}$ and $L \times \bar{L}$. Thus if the irreducible representation $L$ has a character $F_{L}$ this character determines $L$ to within equivalence. Indeed $L$ is so determined by the restriction of $F_{L}$ to any ideal in $Q^{L}(G)$ on which it is not identically zero. It can be shown that $L$ cannot have a character in the sense just described unless it is of the form $n_{1} L^{1} \oplus n_{2} L^{2} \oplus \cdots \oplus n_{k} L^{k} \oplus \cdots$ where the $L^{j}$ are irreducible. When it is, $V^{F_{L}} \simeq L^{1} \times \bar{L}^{1} \oplus \cdots \oplus L^{k} \times \bar{L}^{k} \oplus \cdots$ just as in the finitedimensional case.

The correspondence between certain infinite-dimensional representations and their characters which we have just described can be extended to a much wider class if we give up one-to-oneness and associate representations with characters instead of vice-versa. Let us define a character to be any linear functional $F$ which is defined on a self adjoint ideal $\mathscr{g}_{F}$ in $Q(G)$ and which has the following further properties:

(a) $F(\alpha \beta)=F(\beta \alpha)$ for all $\alpha, \beta$ in $\mathscr{g}_{F}$.

(b) $F\left(\alpha \alpha^{*}\right) \geqq 0$ for all $\alpha$ in $\mathscr{g}_{F}$.

(c) $F$ is a Borel function with respect to the topology which $g_{F}$ inherits from the usual norm topology in $a(G)$. Then $V^{F}$ may be de- 
fined just as above and can be shown ${ }^{3}$ to be a multiplicity free representation of $G \times G$. If $G$ is a type I group there exists a unique measure class in $\hat{G}$ such that $V^{F} \simeq \int_{\hat{G}} L^{s} \times \bar{L}^{s} d \mu(s)$ for any $\mu$ in this measure class. The measure class is concentrated in the set of all $L$ 's for which $F_{L}$ is defined on $\mathscr{g}_{F}$ and there is a unique member of it, $\mu_{0}$ such that $F(\alpha)=\int_{\hat{G}} \mathfrak{C}^{L^{b}}(\alpha) d \mu_{0}(s)$ for all $\alpha$ in $\mathfrak{S}_{F}$. We may think of $F$ as being a character of all representations quasi equivalent to $\int L^{*} d \mu(s)$. Actually there will be many different characters $F$ for a given $V^{F}$. They will correspond one-to-one to certain measures in the measure class of $\mu_{0}$ - those for which $\int X^{L^{s}}(\alpha) d \mu(s)$ exists.

Note that though we have lost one-to-oneness for the correspondence between representations and characters we have achieved a certain uniqueness in direct integral decompositions. Any character on a type I group has a unique representation as an integral of irreducible characters. Thus whenever a canonical character may be selected for a given representation we have, corresponding to it, a well determined element in the measure class in $\hat{G}$ defined by its direct integral decomposition. For example the regular representation of a unimodular group is that defined by the linear functional $f \mu \rightarrow f(e)$ defined for all continuous $f$ with compact support where $\mu$ is Haar measure for the group. The corresponding measure $\hat{\mu}$ in $\hat{G}$ is called the Plancherel measure for $G$ and the formula $f(e)=\int_{\hat{\theta}} \operatorname{Trace}\left(L_{f_{\mu}}^{s}\right) d \hat{\mu}(s)$ is called the Plancherel formula for $G$. When $G$ is commutative $\hat{G}$ is itself a group, $\hat{\mu}$ is just Haar measure in $\hat{G}$ and the Plancherel formula for the translate of $f$ by $x$ becomes the Fourier inversion formula. Of course $\hat{\mu}$ depends upon $\mu$ which is determined only up to a multiplicative constant.

When $G$ is not a type I group there may exist characters $F$ such that $V^{F}$ is irreducible but not of the form $L \times \bar{L}$. The restriction of such a $V$ to $G \times e$ will be a type II primary representation. When the regular representation of $G$ is not of type I the character $f \mu \rightarrow f(e)$ can still be decomposed as above but now some of the components will be associated with type II primary representations instead of irreducible ones. Correspondingly the Plancherel measure must extend over type II primary representations and the Plancherel formula adjusted by a suitable redefinition of $\operatorname{Trace}\left(L_{f_{\mu}}^{s}\right)$. Similar remarks apply to the decomposition of other characters.

Let $L$ and $M$ be irreducible representations of $G$ having characters

${ }^{3}$ Since this was written it has occurred to the author that it is not obvious that the properties (a), (b) and (c) are strong enough to imply that the relevant Hilbert space is separable. In any event one can simply assume this separability as a separate property (d). 
$F^{L}$ and $F^{M}$. Then $L \times M$ has a character whose domain of definition includes all $\alpha \times \beta$ where $\alpha$ and $\beta$ are in the domains of $F^{L}$ and $F^{M}$ respectively. Suppose that this domain includes measures whose supports are in the "diagonal" $\widetilde{G}$ of $G \times G$, i.e. the set of all $x, y$ with $x=y$. Then its restriction to these measures defines a character for $G$. The decomposition of this character defines a unique measure in $\hat{G}$ which we may call the Plancherel measure for the decomposition of the Kronecker product of $L$ and $M$.

Let $G$ be unimodular and let $\mu$ be a Haar measure in $G$. Let $L$ be an irreducible representation of $G$. Then $L$ is equivalent to a subrepresentation of the regular representation if and only if $x \rightarrow\left(L_{x}(\phi) \cdot \psi\right)$ is in $\mathcal{L}^{2}(G, \mu)$ for all $\phi$ and $\psi$ in $\mathscr{H C}(L)$. Moreover if $\left(L_{x}(\phi) \cdot \psi\right)$ is in $\mathscr{L}^{2}(G, \mu)$ for some $\phi$ and $\psi$ with $\phi \neq 0$ and $\psi \neq 0$ then $L$ is equivalent to a subrepresentation of the regular representation. Such representations are said to be square integrable. When $G$ is compact, so that $\mu(G)<\infty$ every irreducible $L$ is square integrable and one has the "orthogonality relations."

$$
\int\left(L_{x}^{1}(\phi) \cdot \psi\right)\left[\left(L_{x}^{2}\left(\phi^{\prime}\right) \cdot \psi^{\prime}\right) d \mu(x)\right]^{-}=0 \text { if } L^{1} \not L^{2}
$$

and

$$
\int\left(L_{x}(\phi) \cdot \psi\right)\left[\left(L_{x}\left(\phi^{\prime}\right) \cdot \psi^{\prime}\right)\right]-d \mu(x)=\frac{\mu(G)}{\operatorname{dim}(L)}\left(\phi \cdot \phi^{\prime}\right)\left[\left(\psi \cdot \psi^{\prime}\right)\right]^{-}
$$

for all $\phi, \psi, \phi^{\prime}$ and $\psi^{\prime}$ in the appropriate Hilbert spaces. Here $\operatorname{dim}(L)$ denotes the dimension of $\mathcal{F C}(L)$. On the other hand the Plancherel measure $\hat{\mu}$ in $\hat{G}$ assigns the measure $\operatorname{dim}(L) / \mu(G)$ to the representation $L$. Thus we may write the second orthogonality relation in the form

$$
\int\left(L_{x}(\phi) \cdot \psi\right)\left[\left(L_{x}(\phi) \cdot \psi\right)\right]-d \mu(x)=\frac{(\phi \cdot \phi)[(\psi \cdot \psi)]}{\hat{\mu}(L)} .
$$

In this form it makes sense whether or not $G$ is compact and both orthogonality relations can be shown to hold for square integrable representations of arbitrary unimodular groups. It is suggestive to think of $\hat{\mu}(L)$ as the finite ratio of the infinite dimension of $\Re(L)$ to the infinite measure of $G$. In particular it makes sense to talk of the ratio of the dimensions of two infinite-dimensional square integrable representations. Amongst examples of noncompact groups with nontrivial square integrable representations are the group of all $2 \times 2$ real matrices of determinant one, certain other noncomplex semi- 
simple Lie groups and certain groups with compact open normal subgroups.

Let $K$ be a compact subgroup of the group $G$. For each irreducible representation $L$ of $G$ we may consider the primary decomposition of the restriction $L^{K}$ of $L$ to $K$. Let $\mathfrak{K}_{d}(L)$ denote the subspace of $\mathfrak{K C}(L)$ on which $L^{K}$ is a multiple of the irreducible representation $d$ of $K$ and let $P_{d}^{L}$ denote the projection on $\mathfrak{F C}_{d}(L)$. Whenever $\mathfrak{K}_{d}(L)$ is finitedimensional we may form the function $x \rightarrow \operatorname{Trace}\left(P_{d}^{L} L_{x}\right)$. It is called the spherical function of $L$ associated with $d$ and denoted by $\phi_{a}^{L}$. If $L$ is finite-dimensional then $\operatorname{Trace}\left(L_{x}\right)=\sum_{d} \phi_{d}^{L}(x)$. In general we may think of $\phi_{d}^{L}$ as being the $d$ component of the "generalized function" which is the character of $L$ and may think of this character as a formal sum of the $\phi_{d}^{L}$. If $\mathfrak{F C}_{d}(L)$ is finite-dimensional for all $L$ and $d$ we shall say that $K$ is a large compact subgroup of $G$. It is easy to see that if $K_{1} \subseteq K_{2}$ and $K_{1}$ is large then $K_{2}$ is also large. It is known that the maximal compact subgroup of the quotient of a semisimple Lie group by its center is large and that a compact subgroup $K$ is large whenever there is a commutative normal subgroup $N$ such that $N K=G$. Many of the special properties of semisimple Lie groups hold for all groups which admit a large compact subgroup. For example such a group is always of type I and every irreducible representation has a character. Moreover there exists a single ideal on which all characters are defined. Each irreducible representation $L$ is determined to within equivalence by any nonvanishing $\phi_{d}^{L}$. For each $d$ the set of all $L$ with $\phi_{d}^{L} \neq 0$ and a given value for $\operatorname{dim} \mathfrak{K}_{d}(L)$ can be given a natural Hausdorff locally compact topology. Thus though the natural topology which one can introduce into $\hat{G}$ makes it into a non-Hausdorff space-even in very simple cases-this space can be written as a countable union of (overlapping) Hausdorff locally compact subspaces.

Let $a^{c}(G)$ denote the center of $a(G)$. If $L$ is an irreducible representation of $G$ then $L_{\alpha}$ commutes with $L_{x}$ for all $x$ in $G$ and all $\alpha$ in $Q^{1}(G)$ so that $L_{\alpha}$ must be a scalar multiple of the identity: $L_{\alpha}=h^{L}(\alpha) I$. Each $h^{L}$ is a homomorphism of the commutative Banach algebra $a^{c}(G)$ into the complex numbers. When $G$ is compact the homomorphism $h^{L}$ determines $L$ to within equivalence. Its restriction to $\mathscr{L}^{1}(G)$ is defined by a bounded function which is a constant multiple of Trace $\left(L_{x}\right)$. For noncompact $G$ this way of defining characters is quite inadequate. For most interesting noncompact groups the conjugate classes are so large that $Q^{c}(G)$ is too small for $L \rightarrow h^{L}$ to be anywhere near to being one-to-one. In fact it is quite often the case that $Q^{c}(G)$ contains only zero. On the other hand when $G$ is a connected Lie 
group one can replace $Q(G)$ by the algebra $\tilde{a}(G)$ of all distributions whose support is the identity of the group. While the center of this algebra is not big enough for $h^{L}$ to determine $L$ to within equivalence one does have the following result. Let $G$ be a semisimple Lie group with a finite center and let $K$ be a maximal compact subgroup. Let $d$ be an irreducible representation of $K$. Let $h$ be a homomorphism of the center of $\tilde{a}(G)$ onto the complex numbers. Then there are at most a finite number of inequivalent irreducible representations $L$ of $G$ such that $P_{a}^{L} \neq 0$ and $h^{L}=h . h^{L}$ is called the infinitesimal character of $L$.

5. Induced representations and systems of imprimitivity. Let $H$ be a closed subgroup of the separable locally compact group $G$ and let $L$ be a unitary representation of $H$. Let $\pi$ denote the natural mapping of $G$ onto the space $G / H$ of all $H$ cosets $H x$ and let $\mathfrak{N}(G / H)$ denote the set of all finite Borel measures $\alpha$ on $G / H$ such that $\alpha(E)=0$ whenever $\pi^{-1}(E)$ has Haar measure zero. We define $\sqrt{ } \alpha_{1} \alpha_{2}$ to be the measure whose Radon-Nikodym derivative with respect to $\alpha_{1}+\alpha_{2}$ is

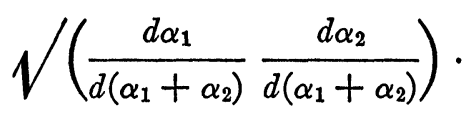

Let $\mathcal{H C}^{L}$ denote the set of all pairs $\alpha, \phi$ where $\alpha$ is in $\Re(G / H)$ and $\phi$ is a Borel function from $G$ to the unit sphere of $\mathcal{K C}(L)$ such that $\phi(\xi x)=L_{\xi}(\phi(x))$ for all $\xi$ in $H$ and all $x$ in $G$. The set on which $\phi(x)$ $=\phi^{1}(x)$ is clearly a union of cosets and we identify $\alpha, \phi$ with $\alpha, \phi^{1}$ whenever $\phi(x)=\phi^{1}(x)$ almost everywhere with respect to $\alpha$. Given two pairs $\alpha_{1}, \phi_{1}$ and $\alpha_{2}, \phi_{2}$ we define their inner product $\left(\alpha_{1}, \phi_{1}: \alpha_{2}, \phi_{2}\right)$ by the formula $\left(\alpha_{1}, \phi_{1}: \alpha_{2}, \phi_{2}\right)=\int_{G / H}\left(\phi_{1}(x) \cdot \phi_{2}(x)\right) d\left(\sqrt{ } \alpha_{1} \alpha_{2}\right)(x)$. This formula makes sense because $\left(\phi_{1}(x) \cdot \phi_{2}(x)\right)$ is constant on the cosets $H x$. With this inner product $\mathcal{F}^{L}$ becomes a Hilbert space; that is, there is a unique way of defining linear combinations so that $\mathfrak{H}^{L}$ is a Hilbert space in the usual sense. For each $x \in G$ let $U_{x}^{L}$ be the operator taking $\alpha, \phi$ into $\alpha_{x}, \phi_{x}$ where $\alpha_{x}(E)=\alpha(E x), \phi_{x}(s)=\phi(s x)$. Then $U_{x}^{L}$ is a one-to-one inner product preserving map of $\mathfrak{F C}^{L}$ on $\mathfrak{F}^{L}$ and hence is a unitary operator. The mapping $x \rightarrow U_{x}^{L}$ is a unitary representation of $G$ which we call the representation of $G$ induced by $L$ and denote by $U^{L}$. In the special case in which $G / H$ admits an invariant measure $\mu$ we may define $\mathfrak{F}^{L}=\mathfrak{H C}\left(U^{L}\right)$ somewhat more perspicuously as the set of all Borel functions $\theta$ from $G$ to $\mathfrak{F C}(L)$ such that $\theta(\xi x)=L_{\xi}(\theta(x))$ for all $\xi, x \in H \times G$ and such that $\int_{G / H}(\theta(x) \cdot \theta(x) d \mu(x))$ $<\infty$. We identify as before and set $\left(\theta_{1}: \theta_{2}\right)=\int_{G / H}\left(\theta_{1}(x) \cdot \theta_{2}(x) d \mu(x)\right)$. The natural correspondence between the two Hilbert spaces is that 
taking $\theta$ into the pair $\alpha_{\theta}, \phi_{\theta}$ where $d \alpha_{\theta} / d \mu=(\theta(x) \cdot \theta(x))$ and $\phi_{\theta}(x)$ $=\theta(x) / \sqrt{ }(\theta(x) \cdot \theta(x))$.

Amongst the more elementary formal properties of the mapping $L \rightarrow U^{L}$ are the following:

(a) $U^{\bar{L}} \simeq\left[U^{L}\right]-$,

(b) $U^{L_{1} \times L_{2}} \simeq U^{L_{1}} \times U^{L_{2}}$ if $H_{1} \subseteq G_{1}, H_{2} \subseteq G_{2}$ and $L_{1}$ and $L_{2}$ are unitary representations of $H_{1}$ and $H_{2}$ respectively,

(c) $U^{M} \simeq U^{L}$ if $H \subset H^{1} \subset G$ and $M$ is the representation of $H^{1}$ induced by $L$,

(d) $U^{L_{1} \oplus L_{2}} \simeq U^{L_{1}} \oplus U^{L_{2}}$ are more generally

$$
U^{\mathcal{S} L^{\lambda} d \mu(\lambda)} \simeq \int U^{L^{\lambda}} d \mu(\lambda)
$$

When $L$ is the one-dimensional identity representation of the subgroup consisting of the identity alone then $U^{L}$ is easily seen to be the regular representation. It follows from this and the "chain rule," (c) above, that $U^{L}$ is the regular representation of $G$ whenever $L$ is the regular representation of $H$.

To a surprisingly large extent the known infinite-dimensional irreducible representations of groups can be thrown into the form $U^{L}$ where $L$ is a one-dimensional representation of a suitable closed subgroup. For this reason it is useful to have criteria for the irreducibility of induced representations. Irreducibility of $L$ is of course necessary but far from sufficient for the irreducibility of $U^{L}$. In the very special case in which there are only a finite number of right $H$ cosets the following necessary and sufficient conditions can be given. If $a$ is an automorphism of $G$ let $L^{a}$ denote the representation of $a^{-1}(H)$ which carries $a^{-1}(h)$ into $L_{h}$ and let $i_{x}$ denote the inner automorphism $y \rightarrow x y x^{-1}$. Then $U^{L}$ is irreducible if and only if for every $x$ not in $H$, $L^{i_{x}}$ and $L$ are disjoint when restricted to $H \cap x^{-1} H x$. This theorem makes sense no matter how many right $H$ cosets there are but is only known to be true in rather special cases. There are also cases in which it is known to be false and one of the more interesting unsolved problems of the theory is that of finding a suitably modified theorem which is true under reasonably general conditions. When $H$ is normal and a type I group the theorem is true as it stands no matter how big $G / H$ is. It then takes the following simplified form: $U^{L}$ is irreducible if and only if $L^{i_{x}}$ and $L$ are inequivalent irreducible representations of $H$ for all $x$ not in $H$. As an example of the application of this last theorem consider the case in which $G$ is the group of all sense preserving rigid motions of the plane and $H$ is the subgroup of all translations. The theorem tells us, more or less immediately, that $U^{L}$ is irreducible 
for every nontrivial irreducible representation $L$ of $H$. Necessary and sufficient conditions for the irreducibility of $U^{L}$ are known when $L$ is finite-dimensional and $G / H$ is countable and useful sufficient conditions are known when $G$ is a Lie group and $L$ is finite-dimensional.

Let $H_{1}$ and $H_{2}$ be closed subgroups of $G$ such that the coset spaces $G / H_{1}$ and $G / H_{2}$ are finite. The irreducibility theorem described above is an immediate consequence of the following theorem about the dimension of $R\left(U^{L}, U^{M}\right)$ where $L$ and $M$ are irreducible representations of $H_{1}$ and $H_{2}$ respectively. For each $x$ and $y$ in $G$ let $J(x, y, L, M)$ denote the dimension of $R\left(L^{i_{x}} \uparrow, M^{i} \uparrow\right)$ where $\uparrow$ applied to each representation denotes its restriction to their common domain $x^{-1} H_{1} x \cap y^{-1} H_{2} y$. Then $J(x, y, L, M)$ depends only upon the double coset $c=H_{1} x y^{-1} H_{2}$ to which $x y^{-1}$ belongs so that we may write $J(c, L, M)$. Moreover $\operatorname{dim} R\left(U^{L}, U^{M}\right)=\sum_{c \in C} J(c, L, M)$ where $C$ is the set of all $H_{1}: H_{2}$ double cosets. Applied to the case in which $H_{2}=G$ this theorem implies the Frobenius reciprocity theorem $\operatorname{dim} R\left(U^{L}, M\right)=\operatorname{dim} R\left(L\right.$, restriction of $M$ to $\left.H_{1}\right)$. In addition it implies a criterion for the equivalence of $U^{L}$ and $U^{M}$ when $L$ and $M$ are both irreducible. When $L$ and $M$ are both finite-dimensional the theorem about $\operatorname{dim} R\left(U^{L}, U^{M}\right)$ is an immediate consequence of a theorem representing $U^{L} \otimes U^{M}$ as a direct sum over $H_{1}: H_{2}$ double cosets on certain other induced representations of $G$. This theorem of Kronecker products is independent of the hypothesis about the finiteness of $G / H_{i}$ and holds at least when there are only countably many $H_{1}: H_{2}$ double cosets. It actually holds more generally if we are willing to replace direct sums by direct integrals. Moreover it is a consequence of an equally general theorem about the restriction of $U^{L}$ to $H_{2}$. Let $C$ denote the set of all $H_{1}: H_{2}$ double cosets and let $\pi$ denote the mapping taking each $x \in G$ into $H_{1} \times H_{2} \in C$. We define a subset $E$ of $C$ to be a Borel set if $\pi^{-1}(E)$ is a Borel set in $G$. If the points of $C$ are separated by countably many Borel sets we shall say that $H_{1}$ and $H_{2}$ are regularly related. The unique measure class in $C$ whose null sets are the sets $E$ such that $\pi^{-1}(E)$ is a null set in $G$ will be called the canonical measure class in $C$. Let $H_{1}$ and $H_{2}$ be regularly related and let $L$ be a unitary representation of $H_{1}$. For each $x \in G$ let ${ }_{x} V$ denote the representation of $H_{2}$ induced by the restriction of $L^{i_{x}}$ to $H_{2} \cap x^{-1} H_{1} x$. Then ${ }_{x} V$ is determined to within equivalence by the double coset $c=\pi(x)$ to which $x$ belongs so that we may write ${ }_{c} V$. Moreover $U^{L}$ restricted to $H_{2}$ is equivalent to $\int_{C}{ }_{c} V d \mu(c)$ where $\mu$ is any measure in the canonical measure class. To deduce the Kronecker product theorem from the restriction theorem we recall that $U^{L} \otimes U^{M}$ may be regarded as the restriction to the diagonal of $G \times G$ of $U^{L} \times U^{M}$ 
and that $U^{L} \times U^{M} \simeq U^{L \times M}$. We leave details to the reader. When $U^{L}$ and $U^{M}$ are infinite dimensional the Kronecker product theorem does not have implications about irreducibility because the operators in $\mathfrak{F C}\left(U^{L}\right) \otimes \mathfrak{H C}\left(U^{M}\right)$ are only the Hilbert-Schmidt operators. However both the restriction theorem and the Kronecker product theorem are useful tools in dealing with induced representations.

The Frobenius reciprocity theorem, classical for finite groups, was stated above only in the very special case in which $G / H$ is a finite set. Much more general formulations are possible. For example let $H$ and $G$ both be groups whose regular representation are of type I. Let $\int_{\hat{G}} F^{s} d \zeta(s)$ be quasi equivalent to the regular representations of $G$ and let $\int \hat{\hat{H}} N^{t} d \eta(t)$ be quasi equivalent to the regular representation of $H$ where $\zeta$ and $\eta$ are finite measures in $\hat{G}$ and $\hat{H}$ respectively. Then there exists a measure $\alpha$ in $\hat{G} \times \hat{H}$, an $\alpha$ measurable function $n$ from $G \times H$ to the countable ordinals, a function $s \rightarrow \beta_{s}$ from $\hat{G}$ to measures in $\hat{H}$ and a function $t \rightarrow \gamma_{t}$ from $\hat{H}$ to measures in $\hat{G}$ such that

(1) $\alpha=\int \beta_{s} d \zeta(s)=\int \gamma_{t} d n(t)$,

(2) $U^{N^{t}} \simeq \int n(s, t) F^{s} d \gamma_{t}(s)$ for almost all $t$,

(3) $F^{s}$ restricted to $H$ is $\simeq \int n(s, t) N^{t} d \beta_{s}(t)$ for almost all $s$.

In particular if one knows the restriction of $F^{s}$ for almost all $s$ then one knows $U^{N^{t}}$ for almost all $t$ and vice versa.

Again let $L$ be a unitary representation of the closed subgroup $H$ of $G$ and for each Borel set $E$ in $G / H$ let $P_{E}$ denote the operator taking the pair $\alpha, \phi$ into the pair $\alpha_{E}, \phi$ where $\alpha_{E}(F)=\alpha(E \cap F)$. It is easy to see that each $P_{E}$ is a projection in $H\left(U^{L}\right)$ and that $E \rightarrow P_{E}$ is a projection-valued measure. For each $x$ in $G$ and each Borel set in $G / H$ we have $U_{x^{-1}}^{L} P_{E} U_{x}^{L}=P_{[E] x}$ where $[E] x$ denotes the transform of $E$ by $x$ under the natural action of $G$ on $G / H$. More generally let Ir be a $G$ space and let $V$ be a unitary representation of $G$. A projection-valued measure $P^{1}$ defined on $\mathscr{N}$ with each $P_{E}^{1}$ an operator in $\mathfrak{H C}(V)$ will be said to be a system of imprimitivity for $V$ based on $\mathfrak{T C}$ provided that $V_{x^{-1}} P_{E}^{1} V_{x}=P^{1}{ }_{[E] x}$ for all $E$ and $x$. The system of imprimitivity $P$ for $U^{L}$ defined above will be called the canonical system of imprimitivity for $U^{L}$. To within equivalence it is the only system of imprimitivity for $U^{L}$ based on $G / H$ and together with $U$ determines $L$ uniquely. More specifically we have the following theorem. Let $H$ be any closed subgroup of the separable locally compact group $G$ and let $V$ be any unitary representation of $G$. Let $Q$ be any system of imprimitivity for $V$ based on the Borel $G$ space $G / H$. Then there exists a unitary representation $L$ of $H$, unique to within equivalence, and a unitary mapping $W$ of $\mathcal{H C}\left(U^{L}\right)$ on $\mathcal{H C}(V)$ such that $W U_{x}^{L} W^{-1}$ $=V_{x}$ for all $x$ in $G$, and $W P_{E} W^{-1}=Q_{E}$ for all Borel subsets of $E$ of 
$G / H$ where $P$ is the canonical system of imprimitivity for $U^{L}$. Moreover $R(L, L)$ is isomorphic to the intersection of $R\left(U^{L}, U^{L}\right)$ with $\mathcal{R}(P, P)$. This theorem, whose proof is rather long, has many applications. We shall refer to it as the imprimitivity theorem.

6. The relationship between $\hat{N}$ and $\hat{G}$ when $N$ is normal in $G$. When the group $G$ has a closed normal subgroup $N$ it is to some extent determined by the two "smaller" groups $N$ and $G / N$ and one can reasonably attempt to describe $\hat{G}$ in terms of $\hat{N}$ and $(G / N)^{\wedge}$. Consider first the special case in which $G$ is isomorphic to $N \times G / N$. The mapping $L, M \rightarrow L \times M$ is one-to-one from $\hat{N} \times(G / N)^{\wedge}$ into $\hat{G}$ and, for any irreducible representation $V$ of $G$, its restrictions to $N \times e$ and $e \times G / N$ are primary. However $V$ will be of the form $L \times M$ if and only if these primary restrictions are both of type I. If either $N$ or $G / N$ is a type I group this will always be the case and we have a natural one-to-one mapping of $\hat{N} \times(\hat{G} / N)$ onto $\hat{G}$.

Consider next the case in which $N$ is commutative and $G$ is a "semidirect product" of $N$ and a second closed subgroup $H$ in the sense that $N \cap H=e$ and $N H=G$. Of course $H$ is isomorphic to $G / N$. Since $N$ is commutative every irreducible representation of $N$ is of the form $\xi \rightarrow \mathfrak{C}(\xi) I$ where $I$ is the identity operator in a one-dimensional space and $x$ is a continuous complex-valued function on $N$ such that $x\left(\xi_{1} \xi_{2}\right)=x\left(\xi_{1}\right) \Upsilon\left(\xi_{2}\right)$ and $\left|x\left(\xi_{1}\right)\right|=\left|x\left(\xi_{2}\right)\right|=1$ for all $\xi_{1}$ and $\xi_{2}$ in $N$. We shall identify the function $\mathscr{X}$ with the corresponding representation. For any automorphism $\alpha$ of $N$ there is a unique automorphism $\alpha^{*}$ of $\hat{N}$ such that $\alpha^{*}(\mathscr{X})(\xi)=\mathscr{X}(\alpha(\xi))$. Let $\alpha_{h}$ denote the automorphism $\xi \rightarrow h \xi h^{-1}$ defined by each $h$ in $H$. By the orbit $\pi(x)$ of $x \in \hat{N}$ we shall mean the set of all $\alpha_{h}^{*}(\mathscr{X})$ with $h \in H$. By the isotropy group $H_{\mathscr{X}}$ of $\mathscr{X}$ we shall mean the subgroup of all $h \in H_{\mathscr{X}}$ such that $\alpha_{h}^{*}(\mathscr{X})=X$. For each pair $\mathscr{X}, L$ consisting of a member $\mathscr{X}$ of $\hat{N}$ and a primary representation $L$ of $H_{\mathscr{X}}$ let $\mathscr{X} L$ denote the primary representation of $N H_{\mathscr{X}}$ which takes $\xi h$ into $\mathscr{X}(\xi) L_{h}$. Then the induced representation $U^{X_{L}}$ is a primary representation of $G$ which is of type $j, j=$ I, II, III if and only if $L$ is of type $j$. Moreover it is irreducible if and only if $L$ is irreducible. The restriction of $U X^{L}$ to $N$ is a multiple of a direct integral of the members of $\pi(x)$ and every primary representation of $G$ whose restriction to $N$ "lies in $\pi(\mathscr{X})$ " in this sense is of the form $U^{X_{L}}$ where $L$ is uniquely determined to within equivalence. If the orbits define a "smooth" equivalence relation in $N$, in the sense that there exists a Borel set which cuts each orbit just once then every primary representation of $G$ is of the form $U^{X_{L}}$ for some $X$ and some primary representation $L$ of $H_{X}$. Thus as $X$ varies over a cross section $C$ for the orbits and, for each $\mathscr{X}, L$ varies over the primary representa- 
tions of $H_{\mathfrak{X}}$, the representations $U^{\mathscr{X} L}$ vary over all primary representations of $G$-each occurring just once. In particular if each $H \mathscr{X}$ is a type I group then $G$ is a type I group. However the orbits need not define a smooth equivalence relation and when they do not there are many irreducible and primary representations in addition to the $U^{X L}$. We shall have more to say about these extra representations later but unfortunately nothing like a complete analysis of them is possible. On the other hand the theorem just quoted makes possible a very complete and satisfying description of all the irreducible representations of numerous noncompact, noncommutative groups. The proof of this theorem leans heavily on the imprimitivity theorem.

Much of what has just been said may be extended to the case in which $N$ need not be commutative and $H$ need not exist provided that $N$ is a type I group. However we may have to replace the representations $L$ of the groups $H_{\Upsilon}$ by so called projective or ray representations of certain subgroups of $G / N$.

A projective unitary representation differs from an ordinary unitary representation in that the requirement that $L_{x y}=L_{x} L_{y}$ is replaced by the requirements $L_{x y}=\sigma(x, y) L_{x} L_{y}$ and $L_{e}=I$ where $\sigma$ is a complex-valued function on $G \times G$. We call $\sigma$ the multiplier of the representation and we call a projective representation whose multiplier is $\sigma$ a $\sigma$-representation. It is easy to see that any multiplier $\sigma$ must be a Borel function from $G \times G$ to the complex numbers of modulus one which satisfies the identity

$$
\sigma(x, y z) \sigma(y, z)=\sigma(x, y) \sigma(x y, z)
$$

and the normalizing condition $\sigma(e, x)=\sigma(x, e)=1$. Conversely given any function $\sigma$ with these properties it can be shown that there exist projective representations with multiplier $\sigma$. If $L$ is a $\sigma$-representation and $\rho$ is a complex-valued Borel function with $\rho(e)=|\rho(x)|=1$ then $x \rightarrow \rho(x) L_{x}$ is a $\sigma^{1}$-representation where

$$
\sigma^{1}(x, y)=\frac{\rho(x) \rho(y)}{\rho(x y)} \sigma(x, y) .
$$

In particular $x, y \rightarrow \rho(x) \rho(y) / \rho(x y)$ is always a multiplier. Multipliers of this form (coboundaries) are said to be trivial and two multipliers whose quotient is trivial are said to be similar. It is clear that the product of two multipliers is a multiplier and that the multipliers form a group under multiplication. The trivial multipliers constitute a subgroup and the corresponding quotient group is called the multiplier group of $G$. It can reduce to the identity but need not do so. The structure of the multiplier group is known for some groups $G$ but much work remains to be done. 
For a fixed $\sigma$ one can develop a theory of the $\sigma$-representations of $G$ which is quite analogous to the theory of the ordinary representations of $G$. In particular practically everything discussed in $\$ \$ 2$, 3 and 5 of these lecture notes may be extended to $\sigma$-representations. Characters for $\sigma$-representations do not seem to have been investigated. On the other hand if $\sigma_{1}$ and $\sigma_{2}$ are nonsimilar multipliers for the same $G$ the theory of the $\sigma_{1}$-representations of $G$ can be as different from the theory of the $\sigma_{2}$-representations of $G$ as the representation theories of two different groups. For example, let $G=H \times \hat{H}$ where $H$ is locally compact and commutative and $\hat{H}$ is its character group. Let $\sigma\left(h_{1}, x_{1} ; h_{2}, x_{2}\right)=x_{1}\left(h_{2}\right)$. Then $\sigma$ is a nontrivial multiplier for the commutative group $G$ and it can be shown that, to within equivalence, $G$ has just one irreducible $\sigma$-representation and that this representation is infinite-dimensional. Commutative groups can also have type II and type III $\sigma$-representations.

To return to the main theme of this section let $N$ be a type I normal subgroup of $G$. For each irreducible unitary representation $W$ of $N$ and each $x$ in $G$ we may form the representation $n \rightarrow W_{x n x^{-1}}=W_{n}^{x}$. To within equivalence $W^{x}$ depends only upon the image of $x$ in $G / N$. Thus $\hat{N}$ is a Borel $G / N$ space in a natural way and we may look at the orbits of the members of $\hat{N}$ under the action of $G / N$. For each $W$ let $H_{W}$ denote the group of all $x$ in $G$ such that $W^{x} \simeq W$. Then $H_{W} \supseteq N$ and $H_{W} / N$ is isomorphic to a subgroup of $G / N$. Just as in the more special case considered above, we may set up a natural oneto-one correspondence between those primary representations of $G$ whose restrictions to $N$ lie in the orbit of $W$ and the primary representations of $H_{W} / N$. However there is one important difference. The relevant primary representation of $H_{W} / N$ may be a $\sigma$-representation for some nontrivial $\sigma$. Since $W^{x} \simeq W$ for each $x \in H_{W}$ there must exist a unitary $V_{x}$ such that $V_{x} W_{n}^{x} V_{x}^{-1}=W_{n}$ for all $n$ in $N$. When $x \in N$ we may take $V_{x}=W_{x}$ but in general $V_{x}$ is arbitrary up to a multiplicative constant of modulus one and there is no canonical way of choosing this constant. Choosing it arbitrarily (but so that $\left(V_{x}(\phi) \cdot \psi\right)$ is always a Borel function) we get a projective representation $x \rightarrow V_{x}$ of $H_{W}$ which reduces on $N$ to $W$. The multiplier for this representation is uniquely determined up to similarity and may be chosen to be of the form $x, y \rightarrow \sigma\left(x^{0}, y^{0}\right)=\sigma^{0}(x, y)$ where $\sigma$ is a multiplier for $H_{W} / N$ and $x \rightarrow x^{0}$ is the canonical mapping of $H_{W}$ on $H_{W} / N$. It is the $(1 / \sigma)$-primary representations of $H_{W} / N$ which correspond to primary representations of $G$ and the one-to-one mapping is that taking $L$ into $U^{v \otimes L^{0}}$ where $L^{0}$ is the $\left(1 / \sigma^{0}\right)$-representation $x \rightarrow L_{x^{0}}$ of $H_{W}$. Just as before, the induced representations $U^{V \otimes L^{0}}$ can be shown to include all pri- 
mary representations when the orbits in $N$ define a smooth equivalence relation.

The fact that we may have to consider projective representations of the $H_{W} / N$ seems at first sight a serious obstacle to using the above described theory inductively to determine the unitary representations of complicated groups in several stages. However it turns out to be possible to carry out the whole discussion starting with projective representations. Thus we can proceed inductively if we consider all possible multipliers for each group concerned.

7. Ergodic systems of imprimitivity and virtual subgroups. Let the separable locally compact group $G$ be a semidirect product of the closed subgroups $N$ and $H$ where $N$ is commutative and normal as in the second paragraph of $\S 6$. Let $V$ be any unitary representation of $G$. Let $V^{0}$ be the unique (to within equivalence) multiplicity free representation quasi equivalent to the restriction $V^{N}$ of $V$ to $N$. By the theory described in $\S 2, V^{0}$ is as associated with a measure class $C_{V}$ in $\hat{N}$ which determines it to within equivalence. For each Borel subset $E$ of $\hat{N}$ there is a unique measure class $C_{V} \cap E$ whose null sets are the sets $F$ such that $F \cap E$ is of measure zero with respect to $C$. Let $P_{E}^{V}$ denote the unique projection in the center of $\Omega\left(V^{N}, V^{N}\right)$ such that $\left(V^{N}\right) P_{E}^{V}$ is quasi equivalent to the representation defined by $C_{V} \cap E$. Then $E \rightarrow P_{E}^{V}$ is a projection-valued measure called the spectral measure of $V$. It is not hard to see that $P^{v}$ is a system of imprimitivity for $V$ and hence also for the restriction $V^{H}$ of $V$ to $H$. Conversely, given a pair consisting of a unitary representation $W$ of $H$ and a system of imprimitivity $P$ for $H$ based on $\hat{N}$, there exists a unique representation $V$ of $G$ such that $V^{H}=W$ and $P^{V}=P$. If $V$ is primary then $P$ is ergodic in the sense that $P_{E}=P_{[E] h}$ for all $h$ in $H$ if and only if $P_{E}=0$ or $P_{(\hat{N}-E)}=0$. Conversely if $P$ is ergodic then $V$ is primary if and only if $R\left(V^{H}, V^{H}\right) \cap R(P, P)$ has a trivial center. Thus, to survey all primary unitary representations of $G$ with a given restriction to $N$ is to survey all unitary representations $W$ of $H$ having a fixed ergodic system of imprimitivity $P$ based on $N$ and such that $\mathbb{R}(W, W)$ $\cap R(P, P)$ has a trivial center.

Let $P$ be a projection-valued measure on $\hat{N}$ which is an ergodic system of imprimitivity for some $W$. Then either $P_{x(x)}=1$ for some orbit $\pi(x)$ or $P_{\pi(x)}=0$ for all orbits $\pi(x)$. In the first case we shall say that $P$ is transitive and in the second that $P$ is strictly ergodic. When $P$ is transitive it may be regarded as based on $\pi(x)$ and hence on the coset space $H / H_{\mathfrak{X}}$. By the imprimitivity theorem the corresponding representations of $H$ correspond one-to-one to the primary 
representations of $H_{X}$ and this is how the imprimitivity theorem is used to deduce the analysis of $\hat{G}$ given in $\S 6$. Now suppose that $P$ is strictly ergodic and let $\phi$ be a vector in $\mathfrak{B C}(P)$ such that $P_{E}(\phi)=0 \mathrm{im}-$ plies $P_{E}=0$. Then $E \rightarrow\left(P_{E}(\phi) \cdot \phi\right)$ is a Borel measure whose null sets are invariant under the action of $H$. Hence the measure class $C$ to which this measure belongs is invariant. It is ergodic in the sense that any Borel set which is invariant under the action of $H$ (modulo sets of measure zero) is either a set of measure zero or the complement of one. It is strictly ergodic in the sense that every orbit has measure zero. Conversely it can be shown (see below for further details) that, every ergodic measure class $C$ arises in this way from an ergodic system of imprimitivity $P$ for a unitary representation $W$ of $H$, that $P$ is strictly ergodic if and only if $C$ is and that $P$ is determined to within equivalence by $C$ and a cardinal number.

It follows from the above that every strictly ergodic invariant measure class in $\hat{N}$ gives rise to a class of "extra" primary (and irreducible) representations of $G$ not covered by the analysis of $\S 6$. To find all primary (and irreducible) representations of $G$ when $\hat{N}$ admits strictly ergodic invariant measure classes one must first find all such measure classes and then for each find all associated primary representations. The second of these difficult problems is essentially the same as that of finding all representations $W$ of $H$ with a fixed strictly ergodic system of imprimitivity $P$ and will be considered to some extent below. Next to nothing can be said at present about the first.

Let $C$ be any ergodic invariant measure class in $\hat{N}$. Let $\mathfrak{F C}_{0}$ be a separable Hilbert space. Let $\mathfrak{F}\left(C, \mathfrak{F}_{0}\right)$ denote the set of all pairs $\alpha, \phi$ where $\alpha$ is a finite Borel measure in $\hat{N}, \phi$ is a Borel function from $\hat{N}$ to the unit sphere of $\mathfrak{H C}_{0}$, and $\alpha(E)=0$ whenever $E$ is a $C$ null set. We identify $\alpha, \phi$ with $\alpha, \phi^{1}$ whenever $\phi(X)=\phi^{1}(X)$ outside of an $\alpha$ null set and make $\mathfrak{F}\left(C, \mathcal{C}_{0}\right)$ into a Hilbert space via the inner product

$$
\left(\alpha_{1}, \phi_{1}: \alpha_{2}, \phi_{2}\right)=\int\left(\phi_{1}(X) \cdot \phi_{2}(X)\right) d \sqrt{ }\left(\alpha_{1} \alpha_{2}\right)(X) .
$$

Just as with the definition of $\mathcal{H C}\left(U^{L}\right)$ in $\S 6$, the action of $H$ on $\hat{N}$ defines a unitary representation $W^{1}$ of $H$ and $E \rightarrow P_{E}$ where $P_{E}(\alpha, \phi)$ $=\alpha_{E}, \phi$ defines an ergodic system of imprimitivity for $W^{1} \cdot P_{E}=0$ if and only if $E$ is a $C$ null set. Let $Q$ be any function from $\hat{N} \times H$ to the unitary operators in $\mathfrak{H}_{0}$ which has suitable measurability properties and satisfies the following identities almost everywhere with respect to $C$.

(*) $Q(x, e) \equiv I$,

(**) $Q\left(x, h_{1} h_{2}\right)=Q\left(x, h_{1}\right) Q\left([x] h_{1}, h_{2}\right)$. 
Let $\widetilde{Q}_{h}$ denote the unitary operator taking $\alpha, \phi$ into $\alpha, Q_{h} \phi$ where $Q_{h} \phi(x)=Q(x, h)(\phi(x))$. Then $h \rightarrow \widetilde{Q}_{h} W_{h}^{1}=W_{h}^{Q}$ is a unitary representation $W^{Q}$ of $H$ having $P$ as system of imprimitivity. Every unitary representation $W$ of $H$ having $P$ as system of imprimitivity is equivalent so some $W^{Q}$ via an equivalence which leaves $P$ fixed. Moreover the pair $P, W^{Q_{1}}$ is equivalent to the pair $P, W^{Q_{2}}$ if and only if there exists a measurable function $x \rightarrow A(\mathfrak{X})$ from $\hat{N}$ to the unitaries in $\mathcal{F}_{0}$ such that for each $h$,

$(* * *) Q^{2}(x, h)=A(x) Q^{1}(x, h) A([x] h)^{-1}$

for $C$ almost all $x$. Finally the commuting algebra intersection $\mathcal{R}(P, P) \cap \mathbb{R}\left(W^{Q}, W^{Q}\right)$ is isomorphic to the algebra $Q_{a}$ of all measurable functions $X \rightarrow B(X)$ from $N$ to the unitaries in $\mathfrak{F}_{0}$ such that for all $h$,

$$
(* * *)^{\prime} \quad B([x] h) Q(x, h) \equiv B(x) Q(x, h)
$$

for $C$ almost all $x$. Of course $B_{1}$ and $B_{2}$ are to be identified when almost everywhere equal. Finding the most general primary representations of $G$ associated with a given ergodic quasi invariant measure class $C$ is thus equivalent to finding the most general solution $Q$ of $\left({ }^{*}\right)$ and $\left({ }^{* *}\right)$ such that $Q_{a}$ has a trivial center-identifying two solutions when $(* * *)$ holds.

When $C$ is concentrated in an orbit $\pi(x)$ then $\left({ }^{* *}\right)$ can be solved and the solutions correlated with the representations of $H_{\otimes}$. In fact this is one way of proving the imprimitivity theorem. On the other hand when $C$ is strictly ergodic we have a well nigh unapproachable problem in noncommutative cohomology theory. One can find explicit solutions of $\left(^{* *}\right)$ - even all of them in certain rather special cases-but deciding when two are equivalent seems very difficult indeed. Yet there is a much closer analogy with the transitive case than at first meets the eye. There is a sense in which the "generalized orbit" defined by $C$ may be associated with a "virtual" subgroup $H_{C}$ of $H$ and the equivalence classes of solutions of $\left({ }^{*}\right)$ regarded as representations of this "virtual" subgroup. While no actual subgroup of $H$ is involved the abstract objects $H_{C}$ behave like subgroups in many ways. One can speak of one virtual subgroup's being contained normally in another, one can consider homomorphisms of one into or onto another, one can form direct products etc. Moreover the theory of the unitary representations of a "virtual" subgroup is very close in structure to the theory of the unitary representations of an actual group. The theory described in $\$ \S 2$ and 3 goes over almost without change though virtual subgroups of type I probably do not exist. One can define characters and Kronecker products and do at least part of the theory of $\$ 4$. In addition one can induce from one 
virtual subgroup to a larger one and carry over much if not all of the theory described in $\$ \S 5$ and 6 . Indeed parts of this theory take on an improved appearance when virtual subgroups are admitted. In the restriction theorem for induced representations, for example, one can omit the assumption that $H_{1}$ and $H_{2}$ are regularly related if one is willing to accept representations induced from virtual subgroups of $\mathrm{H}_{2}$.

The basic idea behind the introduction of the notion of virtual subgroup may be explained as follows. Let $S$ be a standard Borel $G$ space which is transitive in the sense that for each $s_{1}$ and $s_{2}$ in $S$ there exists $x$ in $G$ with $s_{1} x=s_{2}$. For each $s$ in $S$ let $H_{s}$ denote the group of all $x$ in $G$ for which $s x=s$. The groups $H_{s}$ are mutually conjugate closed subgroups and for each $s$ the coset space $G / H_{s}$ is equivalent as a $G$ space (in an obvious sense) to the $G$ space $S$. Since $G / H$ is a transitive standard Borel $G$ space for every closed subgroup $H$ it follows that we have a natural one-to-one correspondence between equivalence classes of transitive standard Borel $G$ spaces on the one hand and conjugate classes of closed subgroups of $G$ on the other. This suggests that notions and theorems about closed subgroups of $G$ should be capable of reformulation so as to be expressed in terms of the corresponding transitive $G$ spaces. It suggests further thatwhen so reformulated-some of these notions and theorems will apply to $G$ spaces which admit an ergodic invariant measure class but are not strictly transitive and hence not equivalent to coset spaces. When they do we may think of them as being about the nonexistent "virtual" subgroups of $G$ which would define the $G$ spaces if only they were transitive. For example $H_{1}$ is continued in some conjugate of $H_{2}$ if and only if there is a Borel map $\theta$ of $G / H_{1}$ on $G / H_{2}$ such that $\theta([s] x)=[\theta(s)] x$ for all $s$ in $G / H_{1}$ and all $x$ in $G$. Correspondingly when such a mapping of the ergodic $G$ space $S_{1}$ on the ergodic $G$ space $S_{2}$ exists we shall say that the virtual subgroup defined by $S_{1}$ is a subgroup of the virtual subgroup defined by $S_{2}$.

As a less trivial example let us consider the meaning to be attached to the notion of a homomorphism of a virtual subgroup into a group. If $\phi$ is a continuous homomorphism of the closed subgroup $H_{1}$ of $G_{1}$ into the group $G_{2}$-then the subgroup $H_{\phi}$ of $G_{1} \times G_{2}$ consisting of all pairs $h, \phi(h)$ with $h \in H_{1}$ closed, uniquely determines $\phi$ and is determined to within conjugacy by the $G_{1} \times G_{2}$ space $\left(G_{1} \times G_{2}\right) / H_{\phi}$. Let $S_{1}$ be a Borel subset of $G_{1}$ which meets each coset $H_{1} x$ just once. Then $S_{1} \times G_{2}$ meets each $H_{\phi}$ coset just once and the natural maps of $S_{1}$ on $G_{1} / H_{1}$ and of $S_{1} \times G_{2}$ on $\left(G_{1} \times G_{2}\right) / H_{\phi}$ are one-to-one. Using these natural maps, $S_{1}$ and $S_{1} \times G_{2}$ become $G_{1}$ and $G_{1} \times G_{2}$ spaces equivalent 
to the coset spaces $G / H_{1}$ and $\left(G_{1} \times G_{2}\right) / H_{\phi}$ respectively. Moreover $(s, y)(x, z)=s x, z^{-1} y \pi(s, x)$ where $s x$ is the $G_{1}$ space action of $x$ on $s$, $\pi$ is a Borel mapping of $S_{1} \times G_{1}$ into $G_{2}$ and $z^{-1} y \pi(s, x)$ is the product of three members of the group $G_{2}$. If $\pi$ is any Borel mapping from $S_{1} \times G_{1}$ into $G_{2}$ and $S_{1}$ is any standard Borel $G$ space then, as is easy to verify $(s, y)(x, z)=s x, z^{-1} y \pi(s, x)$ makes $S_{1} \times G_{2}$ into a Borel $G_{1} \times G_{2}$ space if and only if we have

(†) $\pi(s, e)=e$ for all $s$ in $S_{1}$,

$(\dagger \dagger) \pi\left(s, x_{1} x_{2}\right)=\pi\left(s, x_{1}\right) \pi\left(s x_{1}, x_{2}\right)$

for all $s, x_{1}, x_{2}$ in $S \times G_{1} \times G_{2}$. Moreover if $S_{1}$ is transitive then $S_{1} \times G_{2}$ is transitive and the subgroup leaving $s_{1}, e$ fixed is the set of all $h$, $\phi(h)$ where $s_{1} h=s_{1}$ and $\phi$ is a Borel (and hence continuous) homomorphism of the subgroup $H_{s_{1}}$ into $G_{2}$. Choosing a different cross section $S_{1}^{\prime}$ has the effect of changing $\pi$ to $\pi^{\prime}$ where

$(\dagger \dagger \dagger) \pi^{\prime}(s, x)=a(s) \pi(s, x) a^{-1}(s x)$

and $a$ is a Borel function from $S$ to $G_{2}$. Thus the notion of a Borel homomorphism of $H_{1}$ into $G_{2}$ is equivalent to the notion of an equivalence class of functions satisfying $(\dagger)$ and $(\dagger \dagger)$, the equivalence relation being defined by $(\dagger \dagger \dagger)$. This latter notion does not explicitly involve $H_{1}$ and makes sense for virtual subgroups. Comparing ( $\left.\dagger\right),(\dagger \dagger)$, and $(\dagger \dagger \dagger)$ with $\left({ }^{*}\right),\left({ }^{* *}\right)$, and $\left({ }^{* * *}\right)$ above we see how the unitary representations of $G$ associated with a given ergodic invariant measure class in $N$ may be regarded as being defined by unitary representations of a virtual subgroup of $H$.

When $\mathscr{T}$ is a $G_{1} \times G_{2}$ space each $G_{1} \times e$ orbit is carried into another by each element of $e \times G_{2}$. Thus the set $\mathfrak{M}^{G_{1}}$ of all $G_{1} \times e$ orbits is a $G_{2}$ space in a natural way. When $\Re$ is of the form $\left(G_{1} \times G_{2}\right) / H_{\phi}$, where $\phi$ is a continuous homomorphism of $H_{1}$ into $G_{2}$, then $\mathfrak{T}^{G_{1}}$ will be a standard Borel space in a natural way if and only if $\phi\left(H_{1}\right)=H_{2}$ is closed in $G_{2}$. When this is the case $G_{2} / H_{2}$ is equivalent to $\Re^{G_{1}}$. In other words $H_{2}$ will be a homomorphic image of $H_{1}$ if and only if $G_{2} / H_{2}$ is $\mathbb{M}^{G_{1}}$ for an $\mathscr{T}$ defined in $G_{1} / H_{1} \times G_{2}$ by a $\pi$ satisfying ( $\dagger$ ) and ( $\dagger$ ). A definition for the notion of homomorphism of one virtual subgroup onto another virtual subgroup at once suggests itself. We leave the precise formulation to the reader.

Results in the ergodic theory of flows and measure preserving transformations may be regarded as results about virtual subgroups of the additive group of the real line and of the additive group of the integers respectively. When this point of view is taken some known results receive interesting new interpretations. For example Ambrose has shown how to construct a more or less general ergodic flow from a single measure preserving transformation $T$ on a space $S$ and a posi- 
tive measurable real function $f$ on $S$. This construction is a special case of the above construction of $\mathfrak{N}^{G_{1}}$ from $S_{1}$ and $\pi$. Since $G_{1}$ is infinite cyclic $\pi$ is determined by a single function from $S_{1}$ to $G_{2}$ and $G_{2}$ in this case is the real line. Ambrose's result may thus be looked upon as asserting something about the realizability of virtual subgroups of the real line as homomorphic images of virtual subgroups of the integers. Similar interpretations may be given to Kakutani's notion of induced measures preserving transformations and Anzai's skew product transformations.

Let $C_{1}$ be an ergodic invariant measure class in the standard Borel $G$ space $S_{1}$. Let $S_{2}$ be a second standard Borel $G$ space and let $\theta$ be a Borel map from $S_{1}$ to $S_{2}$ such that $\theta(s x)=\theta(s) x$ for all $x$ and $s$. Let $C_{2}$ be the measure class in $S_{2}$ whose null sets $E$ are the sets such that $\theta^{-1}(E)$ is a $C_{1}$ null set. Then $C_{2}$ is also ergodic and invariant and defines a virtual subgroup of $G$ containing that defined by $S_{1}$ and $C_{1}$. Now an equivalence class of irreducible representations of the virtual subgroup defined by $S_{1}$ and $C_{1}$ determines and is determined by an equivalence class of pairs consisting of a unitary representation $V$ of $G$ and a system of imprimitivity $P$ for $V$ based on $S_{1}$. Given such a pair let $P_{E}^{1}=P_{\theta^{-1}(E)}$ for each Borel set $E$ contained in $S_{2}$. Then $P^{1}$ is a system of imprimitivity for $V$ based on $S_{2}$. The pair $P^{1}, V$ defines an equivalence class of unitary representation of the virtual subgroup defined by $S_{2}$ and $C_{2}$ which depends only upon the equivalence class of the pair $P, V$. In this way we may pass in a canonical way from unitary representations of virtual subgroups to unitary representations of larger virtual subgroups. When the virtual subgroups are actual this passage coincides with that from $L$ to the induced representation $U^{L}$ described in $\$ 5$. This generalization of the inducing process may be defined directly in terms of the functions $Q$. The second form of the definition is the most convenient for many purposes but is rather more complicated.

For further details about virtual subgroups and their unitary representations the reader must be referred to the lecturer's as yet unwritten introductory article on the subject.

8. Nonunitary representations. It is natural to attempt to extend the theory we have been describing by allowing the operators $L_{x}$ to be continuous linear operators in an arbitrary (separable) Banach space or perhaps even in a complete topological vector space. However, very serious obstacles stand in the way of obtaining nearly so complete a theory-even if one sticks to operators in Hilbert space 
and drops only the condition that the $L_{x}$ be unitary. Perhaps the most important of these obstacles is the fact that subrepresentations of nonunitary representations need not have complementary subrepresentations and that correspondingly a nonunitary representation may be reducible without being decomposable as a direct sum. Because of this there is little if any hope of being able to reduce the problem of finding all representations to that of finding all irreducible representations. For example though one knows quite explicitly the finite-dimensional irreducible representations of an $n$-dimensional vector group one does not know the finite-dimensional representations of such a group when $n>1$. The attempt to determine them all leads to complicated and apparently unsolved problems in the ideal theory of polynomial rings. Moreover for any bounded linear operator $A$ in a Banach space one may form $e^{A t}$ for all real $t$ and thus obtain a representation of the real line. It follows that the problem of classifying all representations of the real line includes as a special case that of classifying all bounded linear operators-a problem known to be highly inaccessible even if one considers only operators in Hilbert space. Another difficulty is illustrated by the following. Let $C$ denote the set of all continuous functions with compact support on the separable locally compact group $G$. This space can be given a tremendous number of different norms which are invariant under translation. The completion of $C$ in any of them yields a Banach space representation of $G$ which is analogous to the regular representation and yet not equivalent to it under the obvious notion of equivalence. If we consider these representations as inequivalent our classification problem is enormously complicated by having appended to it a large chunk of the (apparently hopeless) classification problem for Banach spaces. If we do not we must find a new notion of equivalence which identifies them and is satisfactory in other respects. Such a notion has been proposed (and will be discussed below) but has satisfactory properties only in certain important special cases. This difficulty makes itself felt even for irreducible representations as one can see by replacing the regular representation by an irreducible induced representation. Yet another difficulty appears in the very concept of irreducibility. An important property of irreducible unitary representations is that any bounded linear operator in the space of such a representation $L$ may be approximated arbitrarily closely in the strong operator topology by operators of the form $c_{1} L_{x_{1}}+\cdots+c_{n} L_{x_{n}}$. However for nonunitary representations it seems to be very difficult if not impossible to prove that this property is implied by the nonexistence of closed invariant 
subspaces. These irreducible representations which have it are said to be completely irreducible and those which do not-if indeed they exist-are left in an unexplored limbo.

In spite of the discouraging facts recounted above there are cogent reasons for giving at least some attention to nonunitary representations. In studying the irreducible unitary representations of certain groups, especially the semisimple Lie groups, one gets a more natural and well rounded theory by including the completely irreducible nonunitary representations. The unitary ones form a rather artificial subset of the whole. Indeed when one studies the representations of semisimple Lie groups via their Lie algebras it is not always easy to see which Lie algebra representations are going to correspond to unitary representations of the group. A more pressing (but closely related) reason for studying nonunitary representations is that there exist irreducible unitary representations which may be thrown into the form $U^{L}$ where $L$ is a nonunitary one-dimensional representation of a subgroup and (apparently) may not be so expressed for unitary $L$. Of course the definition of $U^{L}$ has to be adapted so that it applies to nonunitary $L$. In addition (as we shall see below) the problem of studying all nondirect sum "extensions" of one irreducible unitary representation by another gives promise of adding interesting new structural features to the "dual object" $\hat{G}$. Finally in so far as one conceives of the theory of infinite-dimensional group representations as being a natural generalization of classical harmonic analysis one hopes ultimately to generalize more than the relatively trivial $\mathfrak{L}^{2}$ theory.

Let $L$ and $M$ be strongly continuous nonunitary representations of the separable locally compact group $G$ in separable Banach spaces $\mathfrak{H C}(L)$ and $\mathfrak{F C}(M)$. It has been proposed (by Naimark) to say that $L$ and $M$ are equivalent if there exists a closed one-to-one linear operator $T$ from a dense invariant subspace of $\mathfrak{K}(L)$ to a dense invariant subspace of $\mathfrak{F C}(M)$ such that $T L_{x}(\phi)=M_{x} T(\phi)$ for all $x$ in $G$ and all $\phi$ in the domain of $T$. We shall call this notion weak equivalence. It is not hard to show that weak equivalence implies equivalence whenever $L$ and $M$ are unitary. On the other hand it is by no means clear that weak equivalence is even an equivalence relation except in special cases. Suppose that $G$ has a large compact subgroup $K$ in the sense of $\$ 4$. For each completely irreducible representation $L$ of $G$ and each irreducible unitary representation $d$ of $K$ one can define the spherical functions $\phi_{d}^{L}$ more or less as in $\S 4$ and it can be shown that $L$ and $M$ are weakly equivalent if and only if $\phi_{d}^{L}=\phi_{d}^{M}$ for all $d$. It follows that weak equivalence is an equivalence relation in this case and that it 
coincides with the "infinitesimal equivalence" of Harish-Chandra when $G$ is a semisimple Lie group with a finite center.

For some purposes it is convenient to reformulate the notion of weak equivalence as follows. Let $X$ be a vector space and let $Y$ be a subspace of the algebraic dual $X^{*}$ of $X$ which is total in the sense that $f(\phi)=0$ for all $f$ in $Y$ implies $\phi=0$. We shall call such a pair a linear system. By an isomorphism of the linear system $X_{1}, Y_{1}$ with the linear system $X_{2}, Y_{2}$ we shall mean a one-to-one linear transformation $A$ from $X_{1}$ onto $X_{2}$ such that $A^{*}$ is one-to-one from $Y_{2}$ onto $Y_{1}$. Here $A^{*}$ is defined by the identity $A^{*}(f)(\phi)=f(A(\phi))$. By an automorphism of $X, Y$ we shall mean an isomorphism of $X, Y$ with itself. By a linear system representation $L$ of $G$ we shall mean a homomorphism $x \rightarrow L_{x}$ of $G$ into the group of automorphisms of a linear system $X, Y$. If $L$ is a linear system representation of $G$ in $X, Y$, and $X_{1}$ and $Y_{1}$ are total subspaces of $X$ and $Y$ invariant under the $L_{x}$ and $L_{x}^{*}$ respectively then restricted to $X_{1}$, the $L_{x}$ define a linear system representation of $G$ in $X_{1}, Y_{1}$. We shall call this second representation a dense contraction of the first. Two linear system representations $L^{1}$ and $L^{2}$ in $X^{1}, Y^{1}$ and $X^{2}, Y^{2}$ respectively will be said to be equivalent if there exists an isomorphism $A$ of $X^{1}, Y^{1}$ with $X^{2}, Y^{2}$ such that $A L_{x}^{1}=L_{x}^{2} A$ for all $x$ in $G$. Now let $L$ be a Banach space representation. Then $L$ is determined to within (strong) equivalence by the corresponding linear system representation in the linear system $\mathfrak{F C}(L)$, [ $\mathfrak{F C}(L)]^{-}$. If $T$ sets up a weak equivalence between $L$ and $M$ then the range and domain of $T$ and $T^{*}$ define dense contractions of these linear system representations and these dense contractions are equivalent. Conversely if $L$ and $M$ have equivalent dense contractions and $A$ sets up the equivalence then $A$ has a closed extension which defines a weak equivalence between $L$ and $M$. Thus $L$ and $M$ are weakly equivalent if and only if they have equivalent dense contractions. In particular a unitary representation is determined to within equivalence by any dense contraction and, given a linear system representation, we may speak unambiguously of its unitary expansion whenever this exists.

Now let $L$ be a Banach space representation of the closed subgroup $H$ of $G$. Let ${ }^{0} X^{L}$ denote the vector space of all Borel functions $f$ from $G$ to $3 C(L)$ which are bounded on compact sets and satisfy the identity $f(\xi x)=L_{\xi} f(x)$ for all $x$ in $G$ and all $\xi \in H$. Let ${ }^{0} Y^{L}$ denote the vector space of all Borel functions $g$ from $G$ to $[\mathfrak{H C}(L)]$ - which are bounded on compact sets and satisfy the identity $g(\xi x)=\bar{L}_{\xi^{-1}}(g(x))$ for all $x$ in $G$ and all $\xi \in H$. Then $g(x)(f(x))$ as a function of $x$ is a Borel function on $G$ which is constant on the $H$ cosets and hence defines a Borel 
function on $G / H$ which is bounded on compact sets. Let ${ }^{00} X^{L}$ denote the set of all pairs consisting of a member $f$ of ${ }^{0} X^{L}$ and a finite measure $\alpha$ of compact support which is absolutely continuous with respect to the unique invariant measure class in $G / H$. Let ${ }^{00} Y^{L}$ be similarly defined with respect to ${ }^{0} Y^{L}$. Given $f, \alpha$ in ${ }^{00} X^{L}$ and $g, \beta$ in ${ }^{00} Y^{L}$ let $(f, \alpha: g, \beta)=\int_{G / H} g(x)(f(x)) d \sqrt{ } \alpha \beta$. Let us identify two members $f, \alpha$ and $f^{\prime}, \alpha^{\prime}$ of ${ }^{00} X^{L}$ if $(f, \alpha: g, \beta)=\left(f^{\prime}, \alpha^{\prime}: g, \beta\right)$ for all $g, \beta$ in $X$ and let us identify two members $g, \beta$ and $g^{\prime}, \beta^{\prime}$ of ${ }^{00} Y^{L}$ correspondingly. Let us denote the sets of equivalence classes by $X^{L}$ and $Y^{L}$ respectively. $(f, \alpha: g, \beta)$ of course depends only upon the equivalence classes of the arguments. It can be shown that there is a unique way of defining the linear operations in $X^{L}$ and $Y^{L}$ so that $X^{L}, Y^{L}$ becomes a linear system in which the linear function defined by $g, \beta$ is $f, \alpha \rightarrow(f, \alpha: g, \beta)$. $X^{L}, Y^{L}$ and $(f, \alpha: g, \beta)$ are invariant under group translation so group translation defines a linear system representation of $G$ in $X^{L}$, $Y^{L}$. If this linear system representation has a unitary expansion we call it the unitary representation of $G$ induced by $L$ and denote it by $U^{L}$. When $L$ itself is unitary it is easy to see that this definition is equivalent to that given in $\$ 5$. For any given $H$ one can raise the following rather interesting question: For which nonunitary irreducible representations of $H$ is $U^{L}$ defined?

Let $L$ and $M$ be Banach space representations of $G$ and let $x \rightarrow A_{x}$ be any strongly continuous function from $G$ to the bounded linear operators from $\mathfrak{H C}(M)$ to $\mathfrak{H C}(L)$ which is a derivation in the sense that it satisfies the identity

(*) $A_{x y}=L_{x} A_{y}+A_{x} M_{y}$

for all $x$ and $y$ in $G$. For each $x$ let $V_{x}^{A}$ be the operator taking $\phi, \psi$ in $\mathcal{H C}(L) \oplus \mathcal{H C}(M)$ into $L_{x}(\phi)+A_{x}(\psi), M_{x}(\phi)$. Then $x \rightarrow V_{x}^{A}$ will be Banach space representation of $G$ having $L$ as a subrepresentation and $M$ as a quotient representation. Moreover it can be shown rather easily that every representation $V$ having $L$ as a subrepresentation and $M$ as a quotient representation is equivalent to some $V^{A}$ provided that the invariant subspace defining the subrepresentation $L$ has a (in general nonvariant) complementary closed subspace. A necessary and sufficient condition that there should exist an invariant complementary closed subspace so that $V \simeq L \oplus M$ is that $A$ be of the form $x \rightarrow L_{x} A_{0}-A_{0} M_{x}$ where $A_{0}$ is some fixed bounded linear operator from $\mathfrak{F C}(M)$ to $\mathfrak{F C}(L)$. Let us call such a derivation exact. The set of all continuous derivations is a vector space and the set of all exact derivations is a subspace of it. The dimension of the quotient space is a measure of the number of essentially distinct extensions of $L$ by $M$ that exist. It can of course be zero-indicating that $L \oplus M$ is the only 
possibility. Let us denote this quotient space by $D(L, M)$. It can be different from zero even when $L$ and $M$ are unitary. When $G$ is commutative it is easy to show that $D(L, M)=0$ whenever $L$ and $M$ are inequivalent and irreducible. On the other hand if $G$ is commutative, $\hat{G}$ is a commutative Lie group, and $L$ is irreducible and defined by $x \in \hat{G}$ then there exists a natural isomorphism of $D(L, L)$ with the complexification of the tangent space to $G$ at $X$. This suggests the possibility of giving some sort of generalized manifold structure to $\hat{G}$ in the general case whenever it is found that $D(L, L) \neq 0$ over a large subset. It would be interesting to know under what circumstances $D(L, L)$ has a finite nonzero dimension.

9. The representations of semisimple Lie groups. In $\S \S 6$ and 7 we discussed a method for finding the irreducible unitary representations of particular groups. This method applies only to groups having proper closed normal subgroups and replaces the given problem by the corresponding one for certain "smaller" groups. It leads to complete results only when at some stage all of the smaller groups are commutative with "known" character groups and various other conditions are satisfied. It must be supplemented by other methods whenever one of the smaller groups is topologically simple in the sense that it admits no proper closed normal subgroup-unless of course this smaller group is commutative. In this section we shall discuss the extent to which it has been possible to determine the irreducible representations of separable locally compact groups which are topologically simple. Let $G$ be such a group. Since the connected component of the identity is a closed normal subgroup $G$ must be either connected or totally disconnected. The study of nondiscrete totally disconnected simple groups has barely begun and we shall say no more about it here. When $G$ is simple and discrete it almost certainly is not of type I unless it is finite and there seems to be little if any hope of giving a satisfying analysis of the irreducible representations of a nontype I group. For some, but not all, of the known simple finite groups it has been found possible to give a complete list of their irreducible representations-or at least the characters of these representations. However there seems to be little in the way of a general theory and the results are too finite and algebraic in character to fit naturally into this account. We are left with the connected ones and it follows from the solution to Hilbert's fifth problem that a connected topologically simple locally compact group is in fact a simple Lie group.

The simple Lie groups are all known, and extensive (though still incomplete) work has been done on the problem of determining their 
irreducible representations, the characters of these and the Plancherel measure in $\hat{G}$. Much of this work can be formulated so that it applies to general semisimple Lie groups, i.e. groups which are locally isomorphic to direct products of connected simple Lie groups. For simplicity of exposition we shall consider only those semisimple Lie groups which have finite centers and no compact components. The irreducible representations of compact simple Lie groups are all finitedimensional and their complete determination is classical. Basic to the general formulation is the Iwasawa structure theorem which may be stated as follows in our special case. Let $G$ be a semisimple Lie group with a finite center and no compact components. Then $G$ has closed connected subgroups $N, D$ and $K$ having the following properties:

(1) $N$ is nilpotent, $D$ is a vector group and $K$ is compact.

(2) The mapping $n, d, k \rightarrow n d k$ from $N \times D \times K$ to $G$ is a homeomorphism onto.

(3) If $A$ is the centralizer of $D$ in $K$ then $S=N D A$ is a semidirect product of $N$ and $D A$.

(4) $K$ is contained in no properly larger compact subgroup of $G$. As an example of the Iwasawa structure theorem it is illuminating to consider the group $\operatorname{SL}(n, C)$ of all $n \times n$ complex matrices of determinant one. Here we may choose $N, D$ and $K$ as follows: $N$ is the group of all matrices in $\mathrm{SL}(n, C)$ which are one on the main diagonal and zero above it. $D$ is the group of all matrices in $\operatorname{SL}(n, C)$ which are real on the main diagonal and zero off of it. $K$ is the group of all unitary matrices in $\operatorname{SL}(n, C)$. With these choices of $N, D$ and $K, A$ comes out to be the group of all matrices in $\operatorname{SL}(n, C)$ which are zero off of the main diagonal and of absolute value one on it and $S$ comes out to be the group of all matrices in $\operatorname{SL}(n, C)$ which are zero above the main diagonal.

Returning to the general case let $W$ be the normalizer of $D$ in $K$. Then $A \subseteq W$ and $W / A$ defines a finite group of automorphisms of $D A$ and hence of $\hat{D} \times \hat{A}$. Let $(\hat{D} \times \hat{A})^{\prime}$ denote the set of all members of $\hat{D} \times \hat{A}$ whose transforms under $W / A$ are all distinct. For each $x \in \hat{D} \times \hat{A}$ let $x^{0}$ denote the one-dimensional representation of $S$, $n d a \rightarrow d a \rightarrow x(d a) I$. It can be shown that the induced unitary representation $U^{X^{0}}$ of $G$ is irreducible whenever $x \in(\hat{D} \times \hat{A})^{\prime}$. It has been conjectured that $U^{X^{0}}$ is irreducible for all $X$ and this has been proved for the classical complex groups and for the group of all $n \times n$ real matrices of determinant one. If $X_{1}$ and $X_{2}$ are in $(\hat{D} \times \hat{A})^{\prime}$ then the irreducible representations $U^{X_{1}^{0}}$ and $U^{X_{2}^{0}}$ are equivalent if and only if $x_{1}$ and $x_{2}$ belong to the same $W / A$ orbit. Let $C$ be a cross section 
for the orbits in $(D \times A)^{\prime}$ under $W / A$. Then $x \rightarrow U^{x^{0}}$ defines a oneto-one mapping $\theta$ of $C$ into $\hat{G}$. In the important special case in which $G$ is a complex analytic Lie group it can be shown that $\hat{G}-\theta(C)$ is of measure zero with respect to the Plancherel measure $\hat{p}$. Thus in this case there is a sense in which the $U^{\mathfrak{X}^{0}}$ comprise "almost all" unitary representations of $G$. Moreover the Plancherel measure can be described without finding any further irreducible representations of $G$. It can be shown that the "transformed measure" $\theta(\hat{\mu})$ such that $\theta(\hat{\mu}(E))=\hat{\mu}(\theta(E))$ is absolutely continuous with respect to the restriction of Haar measure in $\hat{D} \times \hat{A}$ to $C$ and the Radon-Nikodym derivative has been explicitly computed.

Even when $G$ is complex analytic the $U^{\Upsilon^{0}}$ for $\mathscr{X} \in \hat{D} \times \hat{A}$ are known $n o t$ to exhaust the irreducible representations of $G$. Further irreducible representations of $G$ may be constructed in two ways. If $x \in(\hat{D} \times \hat{A})^{\prime}$ it will be possible to extend $x^{0}$ from $S$ to a larger subgroup and consider the representations of $G$ induced by this extended one dimensional representation. For example let $G$ be the group of all $n \times n$ unimodular complex matrics and let $G_{r, n-r}$ denote the subgroup of all matrices which have zeros in the upper right submatrix of $r$ rows and $n-r$ columns. Let $G_{r, n-r}^{\prime}$ denote the subgroup of $G_{r, n-r}$ having zeros in the lower left submatrix of $r$ columns and $n-r$ rows. For each element of $G_{r, n-r}^{\prime}$ we may compute the determinant of the $r \times r$ matrix in the upper left hand corner and obtain in this way a homomorphism of $G_{r, n-r}^{\prime}$ and hence of $G_{r, n-r}$ into the multiplicative group of nonzero complex numbers. Every one-dimensional unitary representation of this last group defines one of $G_{r, n-r}$ which is not in $(\hat{D} \times \hat{A})^{\prime}$ when restricted to $D \times A$. These unitary representations of $G_{r, n-r}$ induce unitary representations of $G$ which can be shown to be irreducible. They belong to what Gelfand and Naimark call the degenerate series. There are analogous degenerate series associated with partitions of $n$ into more than two summands. Returning to the general case again let $X$ be a homomorphism of $D \times A$ into the multiplicative group of all nonzero complex numbers such that $|\mathfrak{X}(d a)| \not \equiv 1$. Let $x^{0}$ be the corresponding nonunitary one-dimensional representation of $S$. Then, as explained in $\S 8, U^{\mathfrak{X}^{0}}$ might exist as the unique unitary expansion of a certain linear system representation. Moreover $U^{\mathfrak{S}^{0}}$ has been shown to be irreducible, when it exists, under conditions analogous to those described above. For the classical complex groups sufficient conditions for the existence of $U^{X^{0}}$ are known and the corresponding irreducible representations of $G$ constitute what Gelfand and Naimark call the supplementary series. Of course one can form $U^{\mathfrak{X}^{0}}$ also when $x^{0}$ is a nonunitary one-dimensional representation of a subgroup 
containing $S$. The representations obtained in this way are said to belong to the supplementary degenerate series. Whether the principal, supplementary, degenerate, and supplementary degenerate series exhaust the irreducible representations of the complex semisimple Lie groups is an open question. Naimark and Berezin have independent papers purporting to show that the answer is yes for the classical complex groups but Berezin's argument seems to have a serious obscurity or two if not an actual gap and the completion of Neumark's argument has yet to be published. For the special case of the group of all $2 \times 2$ complex matrices of determinant one there is no degenerate series and an affirmative answer has been established.

When $G$ is not a complex analytic group much less information is available. In particular $\hat{G}-\theta(C)$ is not of $\hat{\mu}$ measure zero and the representations in $\theta(C)$ are not sufficient to define $\hat{\mu}$ and the Plancherel formula. This fact seems to be closely related to the fact that it is only for the complex groups that almost every group element is conjugate to a member of the maximal commutative subgroup $D A$. For more general semisimple Lie groups one has a finite number (greater than one) of mutually nonconjugate maximal commutative subgroups and each one seems to have associated with it a series of irreducible unitary representations whose $\hat{\mu}$ measure is not zero. However the situation has been completely elucidated only for the group $\operatorname{SL}(n, R)$ of all $n \times n$ real matrices of determinant one and even here one does not know all irreducible unitary representations-only enough so that the rest have Plancherel measure zero. It is only for the group $\operatorname{SL}(2, R)$ that all irreducible representations are known. This group has to within conjugacy one maximal commutative subgroup other than $D A=D$. This is the compact group $K$ of all matrices of the form

$$
\left\|\begin{array}{rr}
\cos \theta & \sin \theta \\
-\sin \theta & \cos \theta
\end{array}\right\| .
$$

For each irreducible representation

$$
\left\|\begin{array}{rr}
\cos \theta & \sin \theta \\
-\sin \theta & \cos \theta
\end{array}\right\| \rightarrow e^{i n \theta}
$$

of this group we have an irreducible representation $V^{n}$ of $G=\operatorname{SL}(2, R)$ defined by an analogue of the inducing process in which Borel functions are replaced by complex analytic functions. The coset space $G / K$ is in a natural way a complex analytic manifold analytically equivalent to the upper half-plane. The representations $V^{n}$ and the members of the principal and supplementary series exhaust the ir- 
reducible representations of $G$. For $\operatorname{SL}(n, R)$ a typical maximal commutative subgroup is defined by a positive integer $k$ with $2 k \leqq n$ and consists of all matrices in $\mathrm{SL}(n, R)$ such that each submatrix

$$
\left\|\begin{array}{cc}
a_{2 j, 2 j} & a_{2 j, 2 j+1} \\
a_{2 j+1,2 j} & a_{2 j+1,2 j+1}
\end{array}\right\|
$$

with $j \leqq k$ is of the form

$$
\lambda_{j}\left\|\begin{array}{rr}
\cos \theta_{j} & \sin \theta_{j} \\
-\sin \theta_{j} & \cos \theta_{j}
\end{array}\right\|
$$

with $\lambda_{j}>0$ and all other nondiagonal elements are zero. Let us denote this commutative subgroup by $D_{k}$. Let $G_{k}$ denote the subgroup of all matrices in $\operatorname{SL}(n, R)$ whose elements are zero except on the main diagonal and in the submatrices

$$
\left\|\begin{array}{cc}
a_{2 j, 2 j} & a_{2 j, 2 j+1} \\
a_{2 j+1,2 j} & a_{2 j+1,2 j+1}
\end{array}\right\|, \quad \text { with } j \leqq k .
$$

Then $G_{k}$ is isomorphic to the direct product of $k$ replicas of $\operatorname{SL}(2, R)$, $n-k-1$ replicas of the multiplicative group of the positive real numbers and $n-1-2 k$ two element groups. Let us denote the product of the noncommutative factors by $G_{k}^{\prime}$ and the product of the commutative factors by $A_{k}$. Then $D_{k}=\left(G_{k}^{\prime} \cap D_{k}\right) A_{k}$ and the general member of $D_{k}$ is defined by a $k$ tuple $n_{1}, \cdots, n_{k}$ of integers and a character $\mathscr{X}$ of $\hat{A}_{k} . n_{1}, \cdots, n_{k}$ and $X$ may in turn be associated with the irreducible representation $V^{n_{1}} \times V^{n_{2}} \times \cdots \times V^{n_{k}} \times x$ of $G_{k}$. Now let $N_{k}$ be the group of all matrices in $\mathrm{SL}(n, R)$ which are one on the main diagonal, zero above it, and have $a_{2 j+1,2 j}=0$ for $j \leqq k$. Then $N_{k} G_{k}$ is a semidirect product of $N_{k}$ and $G_{k}$ and the representation $V^{n_{1}} \times \cdots \times V^{n_{k}} \times x$ of $G_{k}$ defines one of $N_{k} G_{k}$ which is the identity on $N_{k}$. We shall denote it by $L^{n_{1}, n_{2}}, \cdots, n_{k}, X$. The representations

$$
U^{L^{n_{1}, n_{2}}, \cdots, n_{k}, X}
$$

of $\operatorname{SL}(n, R)$ induced by $L^{n_{1}, n_{2}}, \cdots, n_{k}, x_{\text {have been }}$ shown to be irreducible for all $n_{1}, \cdots, n_{k}, x$. In the aggregate they are of nonzero Plancherel measure and constitute the part of the principal series associated with $D_{k}$.

In the general case, Harish-Chandra has indicated a method for reducing the problem of finding a series of irreducible representations for each maximal abelian subgroup $A$ to the case in which $A$ is compact. Moreover he has solved the problem for compact $A$ when the 
maximal compact subgroup $K$ has a one-dimensional center. In this case one obtains a discrete series of square integrable representations quite analogous to the representations $V^{n}$ of $\operatorname{SL}(2, R)$.

There are a number of things that one can say in general about the representations of semisimple Lie groups. The subgroup $K$ of the Iwasawa decomposition is always large in the sense of $\$ 4$ so that one can define spherical functions and one knows that all representations are of type I. Moreover all irreducible representations have characters in the sense of $\S 4$ and these characters may be described by Schwartzian distributions on the group. In many concrete cases these distributions are actually functions and in all cases it has been shown that the distributions reduce to functions-in fact analytic functions -on a dense open subgroup. In addition to the distribution characters one has the infinitesimal characters defined at the end of $\$ 4$. There is a natural mapping of the complex linear functionals on a Cartan subalgebra of the Lie algebra of the group onto the maximal ideals of the center of the infinitesimal group algebra and hence onto the "potential" infinitesimal characters. One knows which of these "potential" infinitesimal characters can actually arise from Banach space representations and those that arise from unitary representations in a Hilbert space form a small subset. In the case of the group of all $2 \times 2$ complex matrices of determinant one the irreducible Banach space representations have all been determined-of course only to within weak equivalence.

10. Applications to quantum mechanics. Let $\Re($ denote the "configuration space" of a classical mechanical system. For example, if the classical system describes the motion of $n$ particles subject to no constraints $\mathbb{T}$ will be an open subset of a $3 n$-dimensional vector space. We shall suppose for the time being that $\mathfrak{T}$ is an open subset of a finite-dimensional vector space $V$ so that the phase space of the system may be taken to be the direct product $\Re \times V^{*}$ where $V^{*}$ is the algebraic dual of $V$. An observable in classical mechanics is a real valued Borel function on $\mathfrak{T} \times V^{*}$. In order to facilitate the passage to quantum mechanics it will be convenient to call a point in $\Re\left(\times V^{*}\right.$ a pure state and use the word state to denote a probability measure in $\mathfrak{T} \times V^{*}$. Here of course we identify a point in $\pi \times V^{*}$ with the probability measure concentrated in that point. The dynamical group of the system is a homomorphism $t \rightarrow U_{t}$ of the additive group of the real line into the group of all one-to-one differentiable maps of $\Re \times V^{*}$ onto itself. It is usually defined by the vector field in $\Re \times V^{*}$ which is its "infinitesimal generator" and is restricted by further requirements which we shall not describe here. If $A$ is an observable, $q, p$ 
a pure state and $t$ a real number, then $A(q, p)$ is the value of the observable $A$ in the pure state $q, p$ and $U_{t}(q, p)$ is the (pure) state of the system $t$ time units after it was in the pure state $q, p$. If $A$ is an observable, $\alpha$ is a state and $E$ is a Borel subset of the real line, then $\alpha\left(A^{-1}(E)\right)$ is the probability that a measurement of $A$ will lead to a value in $E$ when the system is in the state $\alpha$. In classical statistical mechanics one studies states rather than pure state. Clearly the dynamical group $U$ induces a one parameter group of transformations of the space of all states onto itself. The states form a convex set in a natural way and the pure states are just the extreme points of this convex set.

Let $\mathscr{L}_{\mathfrak{c}}$ denote the $\sigma$-Boolean algebra of all Borel subsets of $\Re \times V^{*}$. Then the observable $A$ is completely determined by the mapping $E \rightarrow A^{-1}(E)$ of the $\sigma$-Boolean algebra of all Borel subsets of the real line into $\mathfrak{L}_{c}$, the state $\alpha$ is the mapping $F \rightarrow \alpha(F)$ from $\mathfrak{L}_{\mathfrak{c}}$ to the unit interval and the dynamical group $t \rightarrow U_{t}$ is completely determined by the homomorphism which it defines of the real line into the group of all automorphisms of $\mathfrak{L}_{c}$. Quantum mechanics differs from classical mechanics in that the $\sigma$-Boolean algebra $\mathscr{L}_{0}$ is replaced by the complete lattice $\mathfrak{L}_{\boldsymbol{q}}$ of all projections in a complete separable infinitedimensional Hilbert space $\dddot{F}$. The observables correspond one-to-one to the projection-valued measures on the line (see \$3) and the states to the probability measures on the projections, that is to the functions $\alpha$ from the projections to the unit interval such that $\alpha\left(P_{1}+P_{2}+\cdots\right)$ $=\alpha\left(P_{1}\right)+\alpha\left(P_{2}\right)+\cdots$ whenever $P_{i} P_{j}=P_{j} P_{i}=0$ and such that $\alpha(0)=0, \alpha(I)=1$. If $E \rightarrow Q_{E}$ "is" an observable and $\alpha$ "is" a state then $\alpha\left(Q_{E}\right)$ is the probability that a measurement of the observable will lead to a value in $E$ when the system is in the state $\alpha$. For each unit vector $\phi$ in $\mathcal{F}, P \rightarrow(P(\phi), \phi)$ is a probability measure on the projections which we shall denote by $\alpha_{\phi}$. It has been shown that every probability measure on the projections may be put into the form $\gamma_{1} \alpha_{\phi_{1}}+\gamma_{2} \alpha_{\phi_{2}}+\cdots$ where the $\phi_{j}$ are mutually orthogonal, the $\gamma_{j}$ are positive and $\gamma_{1}+\gamma_{2}+\cdots=1$. The pure states, that is the extreme points in the convex set of all states, are just the $\alpha_{\phi}$. Via the spectral theorem there is a natural one-to-one correspondence between selfadjoint operators and projection-valued measures on the line-hence a natural one-to-one correspondence between observables and selfadjoint operators in $\mathcal{H}$. If $A$ is a selfadjoint operator and $\phi$ a unit vector let $E \rightarrow Q_{E}^{A}$ denote the projection-valued measure corresponding to $A$. Then in the pure state defined by $\phi$ the observable corresponding to $A$ has the probability distribution $E \rightarrow\left(Q_{E}^{A}(\phi), \phi\right)$. This is the basic statistical assertion of quantum mechanics as formulated by von Neumann. 
The dynamical group in quantum mechanics is a continuous homomorphism $t \rightarrow U_{t}$ of the additive group of the real line into the (suitably topologized) group of all automorphisms of $\mathfrak{L}_{\boldsymbol{q}}$. In general an automorphism of $\mathscr{L}_{q}$ will be defined by a unitary or antiunitary operator in $\mathfrak{H C}$ which is unique to within multiplication by a complex constant of modulus one. It can be shown that the $U_{t}$ may all be defined by unitary operators in $\mathcal{H C}$ and that the ambiguous constants may be chosen so that $U_{t}$ is defined by the unitary operator $W_{t}$ where $t \rightarrow W_{t}$ is a unitary representation of the real line. We shall call $W$ the unitary dynamical group of the system. It is uniquely determined by $U$ to within multiplication by $e^{i a t}$ where $a$ is a real constant. Using Stone's theorem we may write $W_{t}=e^{-i H t}$ where $H$ is a selfadjoint operator. When units are suitably chosen (so that Planck's constant is $2 \pi$ ) the observable associated with $H$ is the quantum mechanical analogue of the total energy. $H$ is called the Hamiltonian of the system. Let $\phi$ be a unit vector defining the state of the system at some instant of time. Then $t$ units of time later, the state is described by $W_{t}(\phi)=e^{-i H t}(\phi)$ and when the derivatives exist $d\left(W_{t}(\phi)\right) / d t$ $=-i H W_{t}(\phi)$. This last equation is Schrödinger's equation in abstract form.

Since classical mechanical observables are in a sense "limiting cases" of quantum mechanical observables no one-to-one correspondence is to be expected. On the other hand those classical observables corresponding to functions $A$ on $\mathfrak{T} \times V^{*}$ such that $A(q, p)$ is independent of $p$, i.e. the configuration observables, have unique quantum counterparts and the corresponding selfadjoint operators commute. Let $P_{F}$ be the operator corresponding to the function on IT which is 1 on the Borel subset $F$ of $\mathfrak{N C}$ and zero outside it. Then $F \rightarrow P_{F}$ is a projection-valued measure defined on $9 T$ and for any Borel function $A$ on $\mathfrak{M}$ the corresponding quantum observable is that defined by the projection-valued measure on the line: $E \rightarrow P_{A^{-1}(E)}$. Thus the configuration observables in quantum mechanics are completely specified by giving a projection-valued measure $P$ on the classical configuration space $\mathfrak{T}$. Moreover in so far as there are no observables except those expressible in terms of the configuration observables and their time derivatives the entire system is determined by giving the projection-valued measure $P$ and the unitary dynamical group $W$.

Suppose now that our system is symmetric under the action of a separable locally compact group $G$ and that there is a closed subgroup $G_{0}$ of $G$ such that $\mathfrak{T} /$ is a $G_{0}$ space in a natural way (see $\S 1$ ). The first supposition means that there is a natural homomorphism $\beta \rightarrow T_{\beta}$ of $G$ into the group of automorphisms of $\mathfrak{L}_{q}$ and assuming (for simplic- 
ity) that $G$ is connected we may realize each $T_{\beta}$ by a unitary $S_{\beta}$ in our Hilbert space $\mathfrak{H}$. The $S_{\beta}$ may be chosen so that $\beta \rightarrow S_{\beta}$ is a $\sigma$ representation of $G$ for some (not necessarily trivial) $\sigma$. Moreover it follows from general invariance principles that the $P_{z i}$ and the $S_{\beta}$ must be related as follows: $S_{\beta} P_{E} S_{\beta}^{-1}=P_{[E] \beta}$ for all $\beta$ in $G_{0}$ and all $E \subseteq \mathscr{T}$. In other words $P$ must be a system of imprimitivity for the restriction $S^{0}$ of $S$ to $G_{0}$. This fact has many interesting implications. In the appropriate context and slightly strengthened it is a restatement of the celebrated Heisenberg commutation relations. In what sense this is so will appear below.

For each continuous homomorphism $x \rightarrow \beta_{x}$ of the real line into $G$, $x \rightarrow S_{\beta_{x}}$ is a $\sigma$-representation of the real line which becomes an ordinary unitary representation on multiplying by a suitable complex-valued function of $x$. By Stone's theorem this unitary representation may be put into the form $x \rightarrow e^{i A x}$ where $A$ is a selfadjoint operator. When the $\beta_{x}$ all belong to $G_{0}$, the observable corresponding to $A$ is called the momentum observable associated with the corresponding one parameter group of transformations of $\mathrm{Tr}$. It is unique up to an additive constant. Modulo this additive constant, the momentum observables in quantum mechanics correspond one-to-one to the so called generalized momenta in classical mechanics. If $G_{0}$ contains the group of all translations in physical space then the observable defined by the one parameter subgroup of all translations in a particular direction is called the total linear momentum in that direction. If $G_{0}$ contains the group of all rotations about a particular axis in space then the observable defined by the corresponding one parameter subgroup is called the total angular momentum about that axis.

Suppose now that our system is a one particle system in the sense that $\Re\left(\right.$ is three-dimensional Euclidean space and that $G_{0}$ is the group of all rigid motions in $9 \pi$. Then the projection valued measure $P$ on TC is a transitive system of imprimitivity for $S^{0}$ and we may apply the imprimitivity theorem of $\$ 5$ to obtain rather detailed information about the form of $P$ and $S$ and hence of the configuration and momentum observables. The subgroup $K$ leaving the origin 0 in $\mathfrak{T C}$ fixed is the three-dimensional rotation group and the pair $P, S^{0}$ is determined to within equivalence by a projective representation $L$ of $K$. Using the fact that $G_{0}$ is a semidirect product of $\Re(t$ and $K$ we can rewrite the conclusion of the imprimitivity theorem as follows: He may be realized as the space of all functions $\phi$ from $\mathfrak{T C}$ to $\mathfrak{H C}(L)$ which are square integrable with respect to Lebesgue measure $\mu$ in $\Re$ in such a manner that

(a) $P_{E}$ is multiplication by the characteristic function of $E$ and, 
more generally, for any function $A$ on $\mathfrak{T}$ the corresponding quantum mechanical observable is that associated with the operator $\psi \rightarrow A \psi$.

(b) If $\beta$ is a translation in $G_{0}$ then $S_{\beta}^{0}(\psi)(q)=\psi(q \beta)$. If $\beta \in K$ then $S_{\beta}^{0} \psi(q)=L_{\beta} \psi(q \beta)$.

From (b) we deduce at once that the selfadjoint operator defining the $x$ component of linear momentum is $\psi \rightarrow i \partial \psi / \partial x$ and similarly for $y$ and $z$. (We are of course assuming that Planck's constant is $2 \pi$.) If we add the assumption that there are no observables except those which can be obtained as weak limits of the configuration and momentum observables we may conclude that $L$ is irreducible. As is well known there is to within similarity just one multiplier $\sigma_{0}$ for $K$ other than the identity. Moreover there is to within equivalence just one irreducible projective representation of $K$ of each finite dimension. Those of odd dimension are ordinary representations and those of even dimension are $\sigma_{0}$-representations. It is customary to denote the irreducible $2 j+1$ dimensional projective representation of $K$ by $D_{j}$ where $j=0,1 / 2,1,3 / 2,2, \cdots$. The integer or half integer $j$ is an important invariant of the one particle system. It is called the spin of the particle. Each angular momentum operator can be written naturally in the form $\Omega+\Omega^{\prime}$ where $\Omega$ is independent of $j$ and $\Omega^{\prime}$ has $2 j+1$ proper values varying from $-j$ to $j$ in steps of $1 . \Omega^{\prime}$ is called the spin angular momentum.

To obtain information about the form of the unitary dynamical group $t \rightarrow e^{-i t H}$ we must make further assumptions. Assuming that the spin is zero and that $H$ commutes with the $S_{\beta}^{0}$ it is easy to deduce that $H$ must be of the form $\theta\left(H_{0}\right)$ where $\theta$ is a Borel function and $H_{0}$ is the Laplacian. Similar but more complicated conclusions can be drawn for particles of higher spin. The possibilities for $\theta$ can be sharply reduced by taking into account the nonabsolute character of space. This is done by imbedding $G_{0}$ in the group $G$ of all automorphisms of space-time and demanding (a) that $S^{0}$ be the restriction to $G_{0}$ of some projective representation $S$ of $G$ and (b) that $W$ be the restriction of $S$ to the group of translations in time. When $G$ is the Galilean group we find that $\theta$ must be multiplication by a negative real number which turns out to be $-1 / 2$ times the "mass" of the particle. In particular we recover the classical free particle Schrödinger equation. More generally we may forget about $\theta$ and analyze the possibilities for relativistic free particles of arbitrary spin by studying the possible extensions of $S^{0}$ from $G_{0}$ to $G$ as above. When $G$ is the inhomogeneous Lorentz group we find that there is a one-parameter family of possibilities for each possible spin and that this parameter may be identified as above with the mass of the particle. The same is true when $G$ is the Galilean group. One obtains the usual Schrö- 
dinger equation for a particle in a potential field by assuming that the velocity observables are the same as they are for a free Galilean particle of spin zero.

Now let us change our assumption on TI by supposing that it is an arbitrary finite-dimensional vector space. Let $G_{0}$ be the group of all translations in $\mathfrak{T}$ and suppose that $S^{0}$ is an ordinary representation of $G_{0}$. Each vector $\phi$ in Tr defines a one-parameter subgroup of $G_{0}$ and hence a monentum observable with selfadjoint operator $B_{\phi}$. Each dual vector $f$ in $\mathfrak{T l}^{*}$ is a Borel function on $\mathfrak{T l}$ and hence defines a configuration observable with operator $A_{f}$. The fact that the projection-valued measure on $\mathfrak{T}$ defining the $A_{f}$ is a system of imprimitivity for $S^{0}$ translatesat once into the identity $e^{i A_{f}} e^{i B_{\phi}}=e^{i B_{\phi}} e^{i A_{f}} e^{i f(\phi)}$. If we let $\phi_{1}, \phi_{2}, \cdots, \phi_{n}$ be a basis for $\mathfrak{T l}$ and let $f_{1}, f_{2}, \cdots, f_{n}$ be the dual basis for $9 \pi^{*}$ we get the Heisenberg commutation relations in integrated or Weyl form. To within equivalence these relations have a unique irreducible solution. This theorem, first proved by Stone and von Neumann, is an immediate consequence of the spectral theorem and the imprimitivity theorem. Indeed, modulo the spectral theorem, it is equivalent to the special case of the imprimitivity theorem in which the group is a vector group and acts on itself by way of the group operation.

The interaction of matter with the electromagnetic field is handled in quantum mechanics by regarding the electromagnetic field as a classical mechanical system with an infinite number of degrees of freedom, i.e. having infinite-dimensional configuration and phase spaces. The corresponding quantum system turns out to be equivalent to that for a system of an "indefinite number" of "indistinguishable particles." These particles are identified with the photons of the old quantum theory and in this way the wave-particle paradox is neatly resolved. There is reason to believe that all of the elementary particles of physics - electrons, mesons, etc.-can be best understood as related to some "field" as photons are related to the electromagnetic field. On the other hand the problem of "quantizing a field," that is finding the quantum system corresponding to a classical mechanical system with an infinite-dimensional phase space of a certain kind, is not nearly so well understood as the problem of "quantizing" a system with a finite number of degrees of freedom. When one attempts to adapt the procedures described above one meets all sorts of difficulties and ambiguities corresponding to the complications one encounters on passing from finite-dimensional linear algebra to linear algebra in function spaces and from ordinary to partial differential equations.

It is usual to start by assuming that the Heisenberg commutation 
relations hold (or in dealing with particles of half integral spin that the so called anticommutation relations hold). The Heisenberg commutation relations are easily extended to the infinite-dimensional case-once one has decided what function space to choose for $\mathfrak{T H}$ and what subspace of the algebra dual to use for $\mathfrak{T *}$. However a serious obstacle immediately arises in that these relations now have a continuous infinity of inequivalent irreducible solutions. Moreover the solution which it is appropriate to use in any given problem is not only hard to determine but seems to be sensitive to small changes in the parameters of that problem. For this reason considerable attention has been given to the problem of determining all solutions of these relations. Unfortunately this problem differs from that presented by the finite-dimensional case in that a transitive system of imprimitivity is replaced by a strictly ergodic one--and one is brought face to face with the difficulties discussed in $\$ 7$. Since a vector space acts transitively on itself one might wonder where the strict ergodicity comes from. The answer is that one cannot allow $G_{0}$ to consist of all translations in $\mathfrak{T}$ without reaching a contradiction-due essentially to the fact that an infinite-dimensional vector space cannot have an invariant measure class. One must either weaken the requirement that $P_{E}$ be a projection-valued measure defined on all Borel subsets of $\mathfrak{T}$ or else replace $G_{0}$ by a proper subgroup of the translation in $\Re$. These alternatives are more or less equivalent and both lead to strict ergodicity.

Many other difficulties present themselves and the problem of extending quantum mechanics to infinite-dimensional systems must be considered largely unsolved. Of course the physicists have their heuristic formulations - and these work surprisingly well in certain contexts, at least when certain semi ad hoc "renormalizations" are made to eliminate annoying divergent integrals. However even they are not satisfied with the present form of the theory and a mathematician naturally would like an infinite-dimensional theory as complete and satisfying as the existent finite-dimensional one. Perhaps one can hope that the theory of infinite-dimensional group representations will be instrumental in producing one.

11. Historical and bibliographical remarks. The purpose of the following is to orient the reader in the literature of the material covered in the preceding sections. No attempt has been made to list all relevant papers and it is quite probable that several important ones have been omitted through oversight. We apologize in advance to their authors. 
The material on primary representations and their classification into types I, II, and III is essentially a translation into the language of group representations of the fundamental work of Murray and von Neumann [91] on the dimension theory of operator rings. The "global" theory in which the notion of type is applied to nonprimary representations is correspondingly a translation of work of Dixmier [9] and Kaplansky [68]. This point of view toward the Murray-von Neumann theory was first published by Mackey [80]. Direct integral decompositions were invented by von Neumann in 1938, but his results were not published until 1949 [94]. They were first systematically applied to general group representations by Mautner [83], [84]. However direct integral decompositions were used in special cases by Gelfand and Naimark in 1947 [30] and Kolmogoroff and Adelson-Velskii may be given credit for having anticipated at least a part of the general theory. Various authors have given alternative treatments including Godement [40], Segal [97] and Mackey [79].

The study of the problem of classifying groups according to whether they have only type I representations or not was begun by Mautner in the two papers cited above and continued in later papers [85], [87]. Kaplansky [70] simplified and extended some of Mautner's work. The fact that semisimple Lie groups have only type I representations is due to Harish-Chandra [50]. For the case of finite center, substantial simplifications have been achieved by Godement [44] and Stinespring [100] using a device due to Kaplansky.

The theorem connecting measure classes in $\hat{G}$ and multiplicity free representations of $G$ generalizes the classical Hahn-Hellinger theory for selfadjoint operators in Hilbert space. This generalization is due to Mackey [81] who at the same time defined the Borel structure in $\hat{G}$. The theorem was proved under the dual assumption that $\hat{G}$ has a metrically standard Borel structure and $G$ has only type I representations. In a recent paper [35] Glimm proved that these two assumptions are equivalent, that they imply that $\hat{G}$ is standard and that they are equivalent to a number of other important regularity properties of $G$. Certain extensions and refinements (of the results in the cited Transactions paper) have been found by Ernest [21] and Guichardet [46]. Ernest considers measure classes in the space of all quasi equivalence classes of primary representations and his results have been improved in an unpublished work of Dixmier. We remark that the notions and results of $\$ \S 2$ and 3 apply equally well to the * representations of * algebras and that much of the quoted literature deals with the algebra formulation. 
The introduction of the Borel structure in $\hat{G}$ was inspired by the fact that the natural topology in $\hat{G}$ is seldom Hausdorff-even for quite well-behaved groups-and the fact that only the Borel sets are needed for direct integral theory. On the other hand, the topology is not as bad as it appears to be and there is a growing literature devoted to its study. In addition to a fundamental paper published by Kaplansky in 1951 [69], there are recent contributions due to Fell [22], [23] and Dixmier [19], [20]. The paper of Glimm cited above is also relevant here.

The fact that every separable locally compact group $G$ has a sufficient number of irreducible unitary representations may be proved by applying the direct integral decomposition theorem to the regular representation of $G$. However it was first proved (for not necessarily separable groups) by applying the Krein-Milman theorem to the convex set of all "positive definite functions" on the group. This was done by Gelfand and Raikov [33] and a general theory of positive definite functions and their connections with representations was later published by Godement [38]. In dealing with representations of * algebras instead of groups the positive definite functions are replaced by the positive linear functionals or "states" as they are often called. The theory of states for operator algebras has had an extensive development which we shall not attempt to summarize here. We shall only mention a paper of Kadison [67] in which representations of general operator algebras are described by ideals of null sets in the "pure state space" of the algebra in partial analogy with Mackey's generalization of the Hahn-Hellinger theory.

The general theory of characters as linear functionals on ideals in group algebras described in $\$ 4$ is a mild reworking of ideas of Godement [43], [44]. An earlier and less widely applicable theory is also due to Godement [41]. Godement's two 1954 papers are closely connected with work of Segal on "noncommutative integration" [98] on the one hand and work of Dixmier on "quasiunitary algebras" [10] on the other. Godement did not complete the program begun in his 1954 Annals papers but it has been recently resumed by Guichardet [47]. The abstract Plancherel theorem (for the regular representation) was first found by Segal [95], [96] and Mautner [84].

Orthogonality relations for infinite-dimensional irreducible representations were first found by Bargmann [1] in connection with his studies of $\mathrm{SL}(2, R)$. His results were generalized and thrown into abstract form by Godement [36], [37]. The connection between the orthogonality relations and the Plancherel measure was established for semisimple Lie groups by Harish-Chandra [58]. That this 
connection holds for arbitrary unimodular separable locally compact groups is an unpublished result of Mackey.

The spherical functions $\phi_{d}^{L}$ were introduced by Gelfand [26] in the special case in which $d$ is the one-dimensional identity and $G$ is a Lie group. For $d$ general and $G$ a semisimple Lie group they were first defined by Harish-Chandra [49]. Returning to the case in which $d$ is the one-dimensional identity, some of Gelfand's ideas were generalized to arbitrary locally compact groups by Mautner [86]. A study of the general case was made by Godement [42]. Godement's paper makes quite clear and explicit the role played by large compact subgroups in the theory of semisimple Lie groups and is an excellent introduction to the work of Harish-Chandra. Gelfand's ideas are developed more fully in a joint work with Berezin [5]. The few remarks made in $\$ 4$ are far from doing justice to the extensive theory involved here. The interested reader is referred to the above cited literature for further details.

Infinitesimal characters were introduced by Harish-Chandra [48] and Gelfand [25] but not in terms of the algebra $\tilde{Q}(G)$ as described in $\S 4$. That $\tilde{a}(G)$ is isomorphic to the universal enveloping algebra of Harish-Chandra is quoted in Godement's paper [42] as an unpublished result of $\mathrm{L}$. Schwartz. The theorem stated at the end of $\$ 4$ is also due to Harish-Chandra [50].

The imprimitivity theorem and the definition and properties of induced representations will be found in various papers of Mackey [77], [78], [79], [80]. For finite groups the notion of induced representation is due to Frobenius. This definition and the Frobenius reciprocity theorem were extended to compact groups by Weil in his book. Mackey's definition in the general case was in part inspired by a construction in a note of Godement [39]. The notion of induced representation-at least when the inducing representation is onedimensional-is implicit in the early work of Bargmann [1] and Gelfand and Naimark [30] on the 3- and 4-dimensional Lorentz group. The first analysis of a semidirect product in the infinite case was Wigner's study of the inhomogeneous Lorentz group [103]. The second was Gelfand and Naimark's determination [31] of the irreducible representations of the group of projective transformations of the real line. The general theory of semidirect products which follows from the imprimitivity theorem is described in [77] and [79]. The extension of the semidirect product theory to the case in which $N$ is an arbitrary closed type I normal subgroup of $G$ and in which one deals with projective unitary representations is due to Mackey [82]. Projective representations of the inhomogeneous Lorentz group are 
considered by Wigner [103] and multipliers for projective representations of Lie groups are studied systematically by Bargmann [2].

A considerable development of the theory of induced representations has been given by Bruhat in his thesis [7]. He restricts himself to Lie groups but compensates for this by considering representations in topological vector spaces. Using the distributions of L. Schwartz he is able to obtain useful sufficient conditions for the irreducibility of induced representations. Quite recently [8] he has been able to extend many of his results to arbitrary locally compact groups by way of an extension of the notion of distribution and has applied them to demonstrate the irreducibility of certain representations of totally disconnected analogues of the classical groups. Blattner [6] has improved one of the results in Bruhat's 1956 paper and made several other contributions to the theory. Loomis has given a new proof of the imprimitivity theorem which uses positive definite functions and avoids separability assumptions [76].

There is some material on ergodic systems of imprimitivity in [82] but most of $\S 7$ is to be regarded as a preliminary announcement of work in progress by Mackey.

At an early stage (1951) of his extensive development of the representation theory of semisimple Lie groups (see below), HarishChandra decided, at the suggestion of Chevalley, not to restrict himself to unitary representations. The paper of Godement on spherical function cited above is concerned throughout with Banach space representations and introduces the notion of complete irreducibility. Naimark gave his definition of equivalence for Banach space representations in [92] which at the same time announced a solution of the problem of determining (to within equivalence) all completely irreducible Banach space representations of the Lorentz group. Details are published in his book [93]. The paper of Berezin mentioned in $\S 9$ [4] deals with the problem of extending Naimark's results to all the complex classical groups. The papers of Bruhat cited above deal with nonunitary representations and so do two recent notes of Zhelobenko [104], [105]. Zhelobenko announces some preliminary but provocative results on the classification of reducible nonunitary representations.

In $\S 8$ the statements about linear system representations, the definition of $U^{L}$ when $L$ is nonunitary and the definition of $\mathscr{D}(L, M)$ are unpublished remarks of Mackey. The definition of $D(L, M)$ was in part inspired by a note of Zhelobenko.

The problem of doing non $\mathscr{L}^{2}$ harmonic analysis on noncompact noncommutative groups has barely begun but the important special 
case of the $2 \times 2$ real unimodular group has been extensively studied in three long papers by Mautner and Ehrenpreis [88], [89], [90]. Kunze and Stein [75] have done further work on this group and Kunze [74] has proved a rather general theorem about $\mathfrak{L}^{P}$ Fourier transforms. A note of Gelfand [27] is also relevant here.

The unitary representation theory of semisimple Lie groups began with studies of the $2 \times 2$ real and complex unimodular groups by Bargmann [1] on the one hand and Gelfand and Naimark on the other. Bargmann's paper [1] contained a detailed study of the real case and some indications concerning the complex one. Gelfand and Naimark [30] treated the complex case only. Later they wrote a series of papers extending their results to all of the classical complex groups. This work was published in book form [32] by the Steklov Institute in 1950. Another approach to the Plancherel formula for these groups will be found in a paper by Gelfand and Graev [28]. The cited results on the $n \times n$ real unimodular group with $n>2$ are due to Gelfand and Graev [29]. As with all the results described in $\$ 9$ the lecturer has taken liberties with the original formulations in order to bring out the connection with the theory of induced representations. Graev has undertaken further studies of real simple Lie groups on his own. Most of his results [45] concern the group of all $n \times n$ complex nonsingular matrices which leave invariant an indefinite Hermitian form.

The study of the infinite-dimensional irreducible representations of abstract semisimple Lie groups was begun by Harish-Chandra and has led to a long series of articles [48], [49], . . , [66]. Insofar as possible Harish-Chandra works with the Lie algebra of the group rather than the group itself. Many of his results are theorems about Lie algebras and a large part of his 1953 Transactions paper is devoted to developing the theory necessary to transform results about infinite-dimensional representations of Lie algebras into results about infinite-dimensional representations of Lie groups. The general results cited in $\$ 9$ about infinitesimal and distribution characters and about the Plancherel formula for complex groups are due to HarishChandra and are proved in the Transactions papers. The American Journal papers are devoted to various aspects of the still unsolved problem of finding almost all irreducible representations and the Plancherel measure for the general real semisimple Lie group.

A systematic study of the unitary representations of simply connected nilpotent Lie groups has been undertaken by Dixmier [11], $[12],[14],[15],[16],[17],[18]$. Not only are all such groups of type I but their irreducible representations have distribution characters. Moreover barring a certain exceptional set of Plancherel measure 
zero the irreducible representations are determined by their infinitesimal characters. Various particular cases are examined in detail. Further contributions to the theory of nilpotent groups have been made by Kirillov [71], [72, [73], Takenouchi [101], and Fell [24].

The ideas and viewpoints of $\$ 10$ have occurred in various forms with varying degrees of rigor to a number of people and it would be difficult indeed to attach them to their "true" inventors. We shall content ourselves with two more or less indisputable attributions and a few references to papers where further details may be found. We remark that the purpose of $\$ 10$ is less to give a survey of known results than to give some idea of what quantum mechanics is about and how infinite-dimensional group representations may be applied to it.

The result concerning the form of the general probability measure on the projections is a theorem of Gleason [34]. The relationship between the irreducible unitary representations of the inhomogeneous Lorentz group and the possible elementary particles was first pointed out by Bargmann and Wigner [3].

A treatment of elementary quantum mechanics from the point of view of $\$ 10$ will be found in Harvard lecture notes of Mackey. ${ }^{4}$

The Centre National de la Recherche Scientifique in France has issued a volume containing the proceedings of a conference held in Lille in 1957 and entitled Les problemes mathematiques de la theorie quantique des champs. This volume contains a long survey article by Segal [99] and another by Wightman [102]. These articles together form an excellent introduction to the various attempts to put the quantum theory of fields on a rigorous basis and refer to most of the relevant literature up to that time.

\section{APPENDIX}

\section{(Added June 1963)}

In this appendix I shall mention briefly a number of recent developments but shall make no attempt to be exhaustive. Some of these could have been included in the main text. Others had not been published in the summer of 1961 when the main text was written.

A good deal of recent work has been devoted to the problem of decomposing unitary representations of the form $U^{L}$ (see \$5) where $L$ is a finite-dimensional unitary representation of the closed subgroup $H$ of $G$ and $G$ is a semisimple Lie group with a finite center.

- To be published in October by W. A. Benjamin Inc., New York. 
In most of this work $H$ is either a discrete group such that $G / H$ has a finite invariant measure or the subgroup of fixed elements for some involutary automorphism of $G$. For the case in which $L$ is the onedimensional identity and $H$ is a maximal compact subgroup the theory of $U^{L}$ is very closely related to the theory of spherical functions. Harish-Chandra in [130] and [131] has worked toward finding a Plancherel formula for the decomposition in this case and further results have been announced by Gindinkin and Karpelevich in [127].

In [121], [122] and [123] Gelfand and Graev describe a general method for attacking the problem of decomposing $U^{L}$ when $L$ is the one-dimensional identity and apply it to a number of examples. The method may be formulated quite generally but works effectively only when the subgroup $N$ (the horospherical subgroup) is properly chosen. If $S=G / H$ and $N$ is any closed subgroup of $G$ then a horosphere (with respect to $N$ ) is a subset of $S$ of the form $s x N x^{-1}$ where $s$ and $x$ are arbitrary fixed elements of $S$ and $G$ respectively. $G$ acts in a natural way on the space $S^{N}$ of all horospheres; though not in general transitively. Each horosphere may be equipped with a measure in a natural way as may the space $S^{N}$. If $f$ is a suitably restricted function on $S$ we may integrate it over each horosphere and thus obtain a function $f^{\prime}$ on $S^{N}$. The mapping $f \rightarrow f^{\prime}$ intertwines $U^{L}$ with the representation defined in $\mathfrak{L}^{2}\left(S^{N}\right)$ by the action of $G$ (for simplicity we consider only the special case in which $L$ is the identity). When $N$ is suitably chosen it is usually quite easy to analyze this second representation. Thus the analysis of $U^{L}$ reduces to the analysis of the "Radon transform" $f \rightarrow f^{\prime}$ and its inverse. This is the problem in integral geometry to which the titles of [121] and [122] refer. This problem is discussed at length in [124].

The case in which $H$ is discrete and $G / H$ has finite invariant measure is of special interest because of its connection with problems in number theory and the theory of automorphic functions. When $G / H$ is compact it is not hard to show [125] that $U^{L}$ is a discrete direct sum of irreducible representations each of which occurs with finite multiplicity. When $G / H$ is not compact $U^{L}$ may not be a discrete direct sum of irreducibles but Harish-Chandra has shown [132] that such direct summands as do exist all occur with finite multiplicityat least when $L$ is the one-dimensional identity. His argument requires that $H$ satisfy certain auxiliary conditions too complicated to state here. Gelfand and Pyatetzki-Shapiro [126] give a somewhat different theorem for noncompact $G / H$. Assuming that $L$ is the onedimensional identity and that $H$ satisfies auxiliary conditions related to those of Harish-Chandra they single out a closed invariant sub- 
space $\mathfrak{H C}_{0}$ of $\mathfrak{L}^{2}(G / H)$ (defined by means of compact horospheres) and sketch a proof of the fact that this space is a discrete direct sum of irreducible invariant subspaces. When $G / H$ is compact this subspace coincides with the whole space.

In the theory of $G / H$, when $H$ is discrete, a central role is played by a noncommutative generalization of the Poisson summation formula known as the Selberg trace formula. For the moment let $G$ be a finite group and let $H$ be any subgroup of $G$. Then $U^{L}$ is finitedimensional and hence has a character $x: x(x)=$ Trace $\left(U_{x}^{L}\right)$. Let $\mathfrak{X}(x) \equiv n_{1} x_{1}(x)+n_{2} x_{2}(x)+\cdots+n_{k} x_{k}(x)$ where the $n_{j}$ are positive integers and the $x_{j}$ are the characters of the irreducible constituents of $U^{L}$. Let $x^{0}$ be the character of $L$ and let $x^{00}$ be the function on $G$ which coincides with $x^{0}$ in $H$ and is zero outside of $H$. By a wellknown elementary formula we have $x(x)=(1 / o(H)) \sum_{y \in G} x^{00}\left(y x y^{-1}\right)$. The Selberg trace formula for finite $G$ is obtained by equating the two expressions for $x$, multiplying by an arbitrary complex-valued function $f$ on $G$, summing over $G$ and rearranging the left side. It is

$$
(1 / o(H)) \sum_{y \in G} \sum_{x \in H} f\left(y^{-1} x y\right) \mathfrak{X}^{0}(x)=\sum_{j=1}^{k} n_{j}\left(\sum_{x \in G} f(x) x_{j}(x)\right) .
$$

Note that the inner sum on the left-hand side, as a function of $y$, is a constant on the right $H$ cosets. Thus the left side is really a sum over $G / H$. Once this has been observed it is clear that the trace formula may be stated (though not necessarily proved) when $H$ is any discrete subgroup of a separable locally compact group $G$ such that (a) $G / H$ has a finite invariant measure $\mu$ and (b) $U^{L}$ is a discrete direct sum of irreducible representations each of which has a character and occurs with finite multiplicity. One need only replace $\sum_{x \in G} f(x) x_{j}(x)$ by $\int_{G} f(x) x_{j}(x) d x$ and $(1 / o(H)) \sum_{y \in G}$ by an integral with respect to $\mu$. Proofs under various hypotheses have been given in [125], [146] and [147]. In [146] and [147] it is assumed that $G$ has a large compact subgroup (cf. \$4) and only those functions $f$ are considered which are constant on the right $K$ cosets. Under these circumstances the integrals $\int f(x) x_{j}(x)$ may be expressed in terms of spherical functions and one need make no assumption about the existence of characters for the irreducible constituents of $U^{L}$. In [125] attention is restricted to the case in which $L$ is the onedimensional identity.

Answering two questions raised by Kaplansky, Mackey [141] has shown that a nontype I group can have a type I regular representation and Thoma [148] has shown that a discrete group $G$ cannot be of type I unless it has a commutative normal subgroup $N$ such that 
$G / N$ is finite. Dixmier [111] has shown that the universal covering group of a Lie group need not be of type I even if the group itself is of type I.

The details of Ernest's paper [21] have been published [118] and the "unpublished" supplementary remarks of Dixmier referred to in the main text will be found in [115]. Closely related results will be found in [149]. Further results about the Borel structure in $\hat{G}$ and related spaces will be found in the thesis of Guichardet [129] and in papers of Effros [117] and Naimark [145]. Guichardet considers the Borel structure in the space of all "normal" irreducible representations showing it to be analytic and identical with that derived from a certain natural topology in this space. He also gives an example showing that a group with a complete system of finite-dimensional irreducible representations need not have a smooth dual. Effros is concerned with the Borel structure in the space of primitive ideals of a $C^{*}$ algebra.

The work of Dixmier and Fell on the topology of $G$ has been carried further in [112], [113], [116], [119], and [120]. Fell in particular has studied the continuity properties of the inducing process and Glimm [128] has introduced and studied a topology in the space of all irreducible pairs $P, L$ where $P$ is a system of imprimitivity for the representation $L$ of $G$. The $G$ space on which $P$ is defined is fixed and given in advance. Results close to those in [119] will be found in [150].

Guichardet's reworking of Godement's character theory as announced in [47] is carried out in detail in the first chapter of [129].

Various contributions to the general theory of induced representations have been made by Blattner [106], [107], [108], Kleppner [136], [137] and Moore [144]. [106] concerns an abstract formulation of the analogue of the inducing process in which Borel functions are replaced by functions which are "partially analytic." Kunze [139] and Kobayashi [138] have proved theorems about the irreducibility of such representations in which the inducing representation is onedimensional and the analyticity is complete. In [144] Moore considers a variant of the notion of induced representation in which, roughly speaking, summability replaces square summability. The induced representations defined in this way are not Hilbert space representations but yield a rather elegant version of the Frobenius reciprocity theorem in which one has a linear isometry between the relevant spaces of intertwining operators. This work of Moore has been extended and developed further by Kleppner in [137]. These generalizations of the Frobenius reciprocity theorem go in quite a different direction from those in [80] since an irreducible representa- 
tion $L$ admits a nonzero intertwining operator with a reducible representation $M$ only if $M$ has discrete irreducible components.

The generalized distributions studied by Bruhat in [8] were introduced and studied independently by Maurin [142] and G. I. Kac [134]. Kunze and Stein have continued their work on nonunitary representations in [140].

A start on the problem of determining the irreducible representations of totally disconnected simple groups is made in [8], [109], [110], and [143]. Mautner in [143] develops a theory of spherical functions for $2 \times 2$ unimodular matrix groups over totally disconnected fields using the subgroup of matrices with integer coefficients as compact subgroup. Among other things he obtains the Plancherel formula for the coset space. Bruhat in [109] studies the $n \times n$ case and attempts to prove that the compact group in question is large $(\S 4)$. He is only partially successful and the problem of deciding whether the totally disconnected analogues of the classical groups are type I groups or not remains a challenging open question. In [110] the results of [109] are extended to totally disconnected analogues of the unitary symplectic and orthogonal groups.

Dixmier in [114] gives a complete determination of the irreducible representations of the DeSitter group-correcting and rigorizing earlier formal work of L. H. Thomas and T. D. Newton. He indicates that analogous arguments can be used to deal with all semisimple Lie groups. He also investigates the integrability and square integrability of the representations and in this particular case verifies a conjecture of Harish-Chandra.

Harish-Chandra in [133] announces some results (too involved to quote here) allowing him to associate square integrable representations with every character of every maximal abelian subgroup of a semisimple Lie group with finite center. This accomplishes an essential step in his program of determining the Plancherel formula for general semisimple Lie groups.

The details of the work of Kirillov on nilpotent Lie groups appear in [135]. He shows among other things that the equivalence classes of irreducible unitary representations of a simply connected nilpotent Lie group correspond in a natural one-to-one manner to the orbits of the canonical action of the group on the dual of its Lie algebra. This correspondence has elegant properties with respect to the formation of tensor products and the restriction of representations to subgroups.

\section{BIBLIOGRAPHY}

1. V. Bargmann, Irreducible unitary representations of the Lorents group, Ann. of Math. (2) 48 (1947), 568-640. 
2. - On unitary ray representations of continuous groups, Ann. of Math. (2) 59 (1954), 1-46.

3. V. Bargmann and E. P. Wigner, Group theoretical discussion of relativistic wave equations, Proc. Nat. Acad. Sci. U.S.A. 34 (1948), 211-223.

4. F. A. Berezin, Laplace operators on semi-simple Lie groups, Trudy Moskov. Math. Obšx. 6 (1957), 371-463. (Russian)

5. F. A. Berezin and I. M. Gelfand, Some remarks on the theory of spherical functions on symmetric Riemannian manifolds, Trudy Moskov Mat. Obšc. 5 (1956), 311351. (Russian)

6. R. J. Blattner, On induced representations, Amer. J. Math. 83 (1961), 79-98.

7. F. Bruhat, Sur les représentations induites des groups de Lie, Bull. Soc. Math. France 84 (1956), 97-205.

8. - Distributions sur un groupe localement compact et applications d l'etude des représentations des groupes p-adiques, Bull. Soc. Math. France 89 (1961), 43-75.

9. J. Dixmier, Sur la réduction des anneaux d'opérateurs, Ann. Sci. Ecole. Norm. Sup (3) 68 (1951), 185-202.

10. - Algèbres quasi-unitaires, Comment. Math. Helv. 26 (1952), 275-322.

11. - On unitary representations of nilpotent Lie groups, Proc. Nat. Acad.

Sci. U.S.A. 43 (1957), 985-986.

12. - Sur les représentations unitaires des groupes de Lie nilpotents. II, Bull. Soc. Math. France 85 (1957), 325-388.

13. - Sur les représentations unitaires des groupes de Lie algêbriques, Ann. Inst. Fourier (Grenoble) 7 (1957), 315-328.

14. - Sur les representations unitaires des groupes de Lie nilpotents. III, Canad. J. Math. 10 (1958), 321-348.

15. - Sur les representations unitaires des groupes de Lie nilpotents. I, Amer. J. Math. 81 (1959), 160-170.

16. - Sur les representations unitaires des groupes de Lie nilpotents. IV, Canad. J. Math. 11 (1959), 321-344.

17. - Sur les representations unitaires des groupes de Lie nilpotents. V, Bull. Soc. Math. France 87 (1959), 65-79.

18. - Sur les représentations unitaires des groupes de Lie nilpotents. VI, Canad. J. Math. 12 (1960), 324-352.

19. - Sur les $C^{*}$ algèbres, Bull. Soc. Math. France 88 (1960), 95-112.

20. - Sur les structures boreliennes $d u$ spectre d'une $C^{*}$-algebre, Inst. Hautes Etudes Sci. Publ. Math. 6 (1960), 5-11.

21. J. Ernest, $A$ decomposition theory for unitary representations of locally compact groups, Bull. Amer. Soc. Math. 67 (1961), 385-388.

22. J. M. G. Fell, $C^{*}$ algebras with smooth dual, Illinois J. Math. 4 (1960), 221-230.

23. - The dual spaces of $C^{*}$ algebras, Trans. Amer. Math. Soc. 94 (1960), 365-403.

24. - A new proof that nilpotent groups are CCR, Proc. Amer. Math. Soc. 13 (1962), 93-99.

25. I. M. Gelfand, The center of an infinitesimal group ring, Mat. Sb. (N.S.) 26 (68) (1950), 103-112. (Russian)

26. - Spherical functions in symmetric Riemann spaces, Dokl. Akad. Nauk. SSSR 70 (1956), 5-8. (Russian)

27. - The structure of a ring of rapidly decreasing functions on a Lie group, Dokl.. Akad. Nauk. SSSR 124 (1959), 19-21. (Russian)

28. I. M. Gelfand and M. I. Graev, Analogue of the plancherel formula for the classical groups, Trudy Moskov. Mat. Obšx. 4 (1955), 375-404. (Russian) 
29. - Unitary representations of the real unimodular group (principal nondegenerate series), Izvestiya Akad. Nauk. SSSR 17 (1953), 189-248. (Russian)

30. I. M. Gelfand and M. A. Naimark, Unitary representations of the Lorentz group, Izvestiya Akad. Nauk SSSR 11 (1947), 411-504. (Russian)

31. - Unitary representations of the group of linear transformations of the straight line, Dokl. Akad. Nauk. SSSR 55 (1947), 567-570. (Russian)

32. - Unitary representations of the classical groups, Trudy Mat. Inst. Steklov 36 (1950), 288. (Russian)

33. I. M. Gelfand and D. A. Raikov, Irreducible unitary representations of arbitrary locally bicompact groups, Mat. Sb. (N.S.) 13 (55) (1943), 301-316. (Russian)

34. A. M. Gleason, Measures on the closed subspaces of a Hilbert space, J. Math. Mech. 6 (1957), 885-894.

35. J. Glimm, Type I C* algebras, Ann. of Math. (2) 73 (1961), 572-612.

36. R. Godement, Sur les relations d'orthogonalité de V. Bargmann. I. Résultats preliminaires, C. R. Acad. Sci. Paris 225 (1947), 521-523.

37. - Sur les relations d'orthogonalité de V. Bargmann. II. Demonstration générale, C. R. Acad. Sci. Paris 225 (1947), 657-659.

38. - Les fonctions de type positif et la théorie des groupes, Trans. Amer. Math. Soc. 63 (1948), 1-84.

39. - Sur la transformation de fourier dans les groupes discrets, C. R. Acad. Sci. Paris 228 (1949), 627-628.

40. - Sur la theorie des representations unitaires, Ann. of Math. (2) 53 (1951), 68-124.

41. - Memoire sur la theorie des caractères dans les groupes localement compacts unimodulaires, J. Math. Pures Appl. 30 (1951), 1-110.

42. - A theory of spherical functions. I, Trans. Amer. Math. Soc. 73 (1952), 496-556.

43. - Théorie des caractères. I. Algebres unitaires, Ann. of Math. (2) 59 (1954), 47-62.

44. - Theorie des caracteres. II. Definition et proprietés genérales des caracteres, Ann. of Math. (2) 59 (1954), 63-85.

45. M. I. Graev, Unitary representation of real simple Lie groups, Trudy Moskov. Mat. Obšč. 7 (1958), 335-389. (Russian)

46. A. Guichardet, Sur un problème posé par G. W. Mackey, C. R. Acad. Sci. Paris 250 (1960), 962-963.

47. - Sur les caractères des algèbres de Banach d involution, C. R. Acad. Sci. Paris 252(1961), 2800-2862.

48. Harish-Chandra, On some applications of the universal enveloping algebra of a semi-simple Lie algebra, Trans. Amer. Math. Soc. 70 (1951), 28-96.

49. - Representations of semi-simple Lie groups. II, Proc. Nat. Acad. Sci. U.S.A. 37 (1951), 170-173.

50. - Representations of a semi-simple Lie group on a Banach space. I, Trans. Amer. Math. Soc. 75 (1953), 185-243.

51. - Plancherel formula for complex semi-simple Lie groups, Proc. Nat. Acad. Sci. U.S.A. 37 (1951), 813-818.

52. - Representations of semi-simple Lie groups. II, Trans. Amer. Math. Soc. 76 (1954), 26-65.

53. - Representations of semi-simple Lie groups. III, Trans. Amer. Math. Soc. 76 (1954), 234-253.

54. - The Plancherel formula for complex semi-simple Lie groups, Trans. Amer. Math. Soc. 76 (1954), 485-528. 
55. - On the characters of a semi-simple Lie group, Bull. Amer. Math. Soc. 61 (1955), 389-396.

56. - Representations of semi-simple Lie groups. IV, Amer. J. Math. 77 (1955), 743-777.

57. - Representations of semi-simple Lie groups, V, Amer. J. Math. 78 (1956), 1-41.

58. — Representations of semi-simple Lie groups, VI, Amer. J. Math. 78 (1956), 564-628.

59. - The characters of semi-simple Lie groups, Trans. Amer. Math. Soc. 83 (1956), 98-163.

60. - On a lemma of F. Bruhat, J. Math. Pures Appl. (9) 35 (1956), 203-210.

61. - Differential operators on a semi-simple Lie algebra, Amer. J. Math. 79 (1957), 87-120.

62. - Fourier transforms on a semi-simple Lie algebra. I, Amer. J. Math. 79 (1957), 193-257.

63. - Fourier transforms on a semi-simple Lie algebra, II, Amer. J. Math. 79 (1957), 653-686. 760.

64. ——, A formula for semi-simple Lie groups. Amer. J. Math. 79 (1957), 733-

65. - Spherical functions on a semi-simple Lie group. I, Amer. J. Math. 80 (1958), 241-310.

66. —_ Spherical functions on a semi-simple Lie group. II, Amer. J. Math. 80 (1958), 553-613.

67. R. V. Kadison, Unitary invariants for representations of operator algebras, Ann. of Math. (2) 66 (1957), 304-379.

68. I. Kaplansky, Projections in Banach algebras, Ann. of Math. (2) 53 (1951), 235-249.

69. - The structure of certain operator algebras, Trans. Amer. Math. Soc. 70 (1951), 219-255.

70. — Group algebras in the large, Tobhoku Math. J. 3 (1951), 249-256.

71. A. A. Kirillov, Induced representations of nilpotent Lie groups, Dokl. Akad. Nauk SSSR 128 (1959), 886-889. (Russian)

72. ___ On unitary representations of nilpotent Lie groups, Dokl. Akad. Nauk SSSR 130 (1960), 966-968. (Russian)

73. - Unitary representations of nilpotent Lie groups, Dokl. Akad. Nauk SSSR 138 (1961), 283-284. (Russian)

74. R. A. Kunze, $L_{p}$ Fourier transforms on locally compact unimodular groups, Trans. Amer. Math. Soc. 89 (1958), 519-540.

75. R. A. Kunze and E. M. Stein, Uniformly bounded representations and harmonic analysis on the $2 \times 2$ real unimodular group, Amer. J. Math. 82 (1960), 1-62.

76. L. H. Loomis, Positive definite functions and induced representations, Duke Math. J. 27 (1960), 569-580.

77. G. W. Mackey, Imprimitivity for representations of locally compact groups. I, Proc. Nat. Acad. Sci. U.S.A. 35 (1949), 537-545. 592.

78. —- On induced representations of groups, Amer. J. Math. 73 (1951), 576-

79. - Induced representations of locally compact groups. I, Ann. of Math. (2) 55 (1952), 101-139.

80. - Induced representations of locally compact groups. II, Ann. of Math. (2) 58 (1953), 193-221. 
81. - Borel structure in groups and their duals, Trans. Amer. Math. Soc. 85 (1957), 134-165.

82. - Unitary representations of group extensions. I, Acta Math. 99 (1958), $265-311$.

83. F. I. Mautner, Unitary representations of locally compact groups. I, Ann. of Math. (2) 51 (1950), 1-25.

84. , Unitary representations of locally compact groups. II, Ann. of Math. (2) 52 (1950), 528-556.

85. - The structure of the regular representation of certain discrete groups, Duke Math. J. 17 (1950), 437-441.

86. - Fourier analysis and symmetric spaces, Proc. Nat. Acad. Sci. U.S.A. 37 (1951), 529-533.

87. - The regular representation of a restricted direct product of finite groups, Trans. Amer. Math. Soc. 70 (1951), 531-548.

88. F. I. Mautner and L. Ehrenpreis, Some properties of the Fourier transform on semi-simple Lie groups. I, Ann. of Math. (2) 61 (1955), 406-439.

89. - Some properties of the Fourier transform on semi-simple Lie groups. II, Trans. Amer. Math. Soc. 84 (1957), 1-55.

90. - Some properties of the Fourier transform on semi-simple Lie groups. III, Trans. Amer. Math. Soc. 90 (1959), 431-484.

91. F. J. Murray and J. von Neumann, On rings of operators, Ann. of Math (2) 37 (1936), 116-229.

92. M. A. Naimark, On irreducible linear representations of the proper Lorentz group, Dokl. Akad. Nauk SSSR 97 (1954), 969-972. (Russian)

93. - Linear representations of the Lorentz group, 376 pp. Fizmatgiz, Moscow, 1958. (Russian)

94. J. von Neumann, On rings of operators. Reduction theory, Ann. of Math (2) 50 (1949), 401-485.

95. I. E. Segal, The two-sided regular representation of a unimodular locally compact group, Ann. of Math. (2) 51 (1950), 293-298.

96. - An extension of Plancherel's formula to separable unimodular groups, Ann. of Math. (2) 52 (1950), 272-292.

97. - Decomposition of operator algebras. I and II, Mem. Amer. Math. Soc. No. 9 (1952), $67+66$ pp.

98. - A non-commutative extension of abstract integration, Ann. of Math (2) 57 (1953), 401-457.

99. - Caracterisation mathematique des observables en theorie quantique des champs et ses conséquences pour la structure des particules libres, Report of Lille conference on quantum fields, C.N.R.S., Paris, 1959, pp. 57-163.

100. W. F. Stinespring, A semi-simple matrix group is of type I, Proc. Amer. Math. Soc. 9 (1958), 965-967.

101. O. Takenouchi, Sur la facteur representation d'un group de Lie resoluble de type E, Math. J. Okayama Univ. 7 (1957), 151-161.

102. A. S. Wightman, Quelques problemes mathematiques de la theorie quantique relativiste, Report of Lille conference on quantum fields, C.N.R.S., Paris, 1959, pp. 1-35.

103. E. Wigner, On unitary representations of the inhomogeneous Lorentz group, Ann. of Math. (2) 40 (1939), 149-204.

104. D. P. Zhelobenko, A description of a certain class of Lorentz group representations, Dokl. Akad. Nauk SSSR 125 (1958), 586-589. (Russian) 
105. - Linear representations of the Lorentz group, Dokl. Akad. Nauk SSSR 126 (1959), 482-485. (Russian)

\section{BibliogRaphy (SUPPLEMENT)}

106. R. J. Blattner, On induced representations. II. Infinitesimal induction, Amer. J. Math. 83 (1961), 499-512.

107. - On a theorem of G. W. Mackey, Bull. Amer. Math. Soc. 68 (1962), 585-587.

108. - A theorem on induced representations, Proc. Amer. Math. Soc. 13 (1962), 881-884.

109. F. Bruhat, Sur les representations de groupes classiques p-adiques. I, Amer. J. Math. 83 (1961), 321-338.

110. - Sur les représentations de groupes classiques p-adiques. II, Amer. J. Math. 83 (1961), 343-368.

111. J. Dixmier, Sur le revêtment universel d'un groupe de Lie de type I, C. R. Acad. Sci. Paris 252 (1961), 2805-2806.

112. - Points séparés dans le spectre d'une C*-algêbre, Acta Sci. Math. Szeged 22 (1961), 115-128.

113. - Points isolés dans le dual d'un groupe localement compact, Bull. Soc. Math. France 85 (1961), 91-96.

114. - Représentations integrables du groupe de deSitter, Bull. Soc. Math. France 89 (1961), 9-41.

115. - Dual et quasi dual d'une algèbre de Banach involutive, Trans. Amer. Math. Soc. 107 (1963), 278-283.

116. J. Dixmier et P. Bernat, Sur le dual d'un groupe de Lie, C. R. Acad. Sci. Paris 250 (1960), 1778-1779.

117. E. Effros, $A$ decomposition theory for representations of $C^{*}$-algebras, Trans. Amer. Math. Soc. 107 (1963), 83-106.

118. J. Ernest, $A$ decomposition theory for unitary representations of locally compact groups, Trans. Amer. Math. Soc. 104 (1962), 252-277.

119. J. M. G. Fell, The structure of algebras of operator fields, Acta. Math. 106 (1961), 233-280.

120. - Weak containment and induced representations of groups, Canad. J. Math. 14 (1962), 237-268.

121. I. M. Gelfand, Integral geometry and its relation to the theory of representations, Uspehi Mat. Nauk 15 (1960), 155-164. (Russian)

122. I. M. Gelfand and M. I. Graev, Geometry of homogeneous spaces, representations of groups in homogeneous spaces and related questions of integral geometry, Trudy Moskov Mat. Obšc. 8 (1959), 321-390. (Russian)

123. - Resolution of Lorentz group representations into irreducible representations on spaces of functions defined on symmetrical spaces, Dokl. Akad. Nauk SSSR 127 (1959), 250-253. (Russian)

124. I. M. Gelfand and N. Y. Vilenkin, Integral geometry and connections with questions in the theory of representations, Moscow, 656 pp., Fizmatgiz, 1962.(Russian)

125. I. M. Gelfand and I. Pyatetzki-Shapiro, Theory of representations and theory of automorphic functions, Uspehi Mat. Nauk 14 (1959), 171-194. (Russian)

126. - Unitary representations in homogeneous spaces with discrete stationary subgroups, Dokl. Akad. Nauk SSSR 147 (1962), 17-20. (Russian)

127. S. G. Gindikin and F. I. Karpelevic, Plancherel measure of Riemannian 
symmetric spaces of non positive curvature, Dokl. Akad. Nauk 145 (1962), 252-255. (Russian)

128. J. Glimm, Families of induced representations, Pacific J. Math. 12 (1962), $885-911$.

129. A. Guichardet, Caractères des algebres de Banach involutives, Ann. Inst. Fourier (Grenoble) 13 (1962), 1-81.

130. Harish-Chandra, Spherical functions on a semisimple Lie group. I, Amer. J. Math. 80 (1958), 241-310.

131. - Spherical functions on a semisimple Lie group. II, Amer. J. Math. 80 (1958), 553-613.

132. - Automorphic forms on a semisimple Lie group, Proc. Nat. Acad. Sci. U.S.A. 45 (1959), 570-573.

133. - Invariant eigendistributions on semisimple Lie groups, Bull. Amer. Math. Soc. 69 (1963), 117-123.

134. G. I. Kac, Generalized functions on a locally compact group and decompositions of unitary representations, Trudy Moskov Mat. Obš. 10 (1961), 3-40. (Russian)

135. A. A. Kirillov, Unitary representations of nilpotent Lie groups, Uspehi Mat. Nauk 106 (1962), 57-110. (Russian)

136. A. Kleppner, The structure of some induced representations, Duke Math. J. 29 (1962), 555-572.

137. - Intertwining forms for summable induced representations, (to appear).

138. S. Kobayashi, On automorphism groups of homogeneous complex manifolds, Proc. Amer. Math. Soc. 12 (1961), 359-361.

139. R. A. Kunze, On the irreducibility of certain multiplier representations, Bull. Amer. Math. Soc. 68 (1962), 93-94.

140. R. A. Kunze and E. M. Stein, Uniformly bounded representations. II. Analytic continuation of the principal series of representations of the $n \times n$ complex unimodular group, Amer. J. Math 83 (1961), 723-786.

141. G. W. Mackey, Induced representations and normal subgroups, Proc. Internat. Sympos. Linear Spaces, Jerusalem pp. 319-326, Pergamon, Oxford, 1960.

142. K. Maurin, Distributionen auf Yamabe-Gruppen. Harmonische Analyse auf einer Abelschen l.k. Gruppe, Bull. Acad. Polon. Sci. Sér.Sci. Math. Astronom. Phys. 9 (1961), 845-850.

143. F. I. Mautner, Spherical functions aver p-adic fields, Amer. J. Math. 80 (1958), 441-457.

144. C. C. Moore, On the Frobenius reciprocity theorem for locally compact groups, Pacific J. Math. 12 (1962), 359-365.

145. M. A. Naimark, Decomposition into factor representations of unitary representations of locally compact groups, Sibirsk. Mat. Ž. 2 (1961), 89-99. (Russian)

146. A. Selberg, Harmonic analysis and discontinuous groups in weakly symmetric Riemannian spaces with applications to Dirichlet series, J. Indian Math. Soc. 20 (1956), 47-87.

147. T. Tamagawa, On Selberg's trace formula, J. Fac. Sci. Univ. Tokyo Sect. I 8 (1960), 363-386.

148. E. Thoma, Über unitare Darstellungen abzahlbarer, diskreter Gruppen, (to appear).

149. M. Takesaki, On some representations of $C^{*}$ algebras, Tôhoku Math. J. 15 (1963), 79-95.

150. J. Tomiyama, Topological representation of $C^{*}$ algebras, Tôhoku Math. J. 14 (1962), 187-204.

HARVARD UNIVERSITY 